Supporting Information for

\title{
Building endogenous gene connections through RNA self-assembly controlled CRISPR/Cas9 function
}

Jiao Lin ${ }^{1}$, Wei-Jia Wang ${ }^{1}$, Yang Wang ${ }^{1}$, Yan Liu ${ }^{1}$ and Liang $\mathrm{Xu}^{1 *}$

${ }^{1}$ MOE Key Laboratory of Bioinorganic and Synthetic Chemistry, School of Chemistry, Sun Yat-Sen University, Guangzhou, 510275, China.

*E-mail:xuliang33@mail.sysu.edu.cn

\section{Table of Contents}

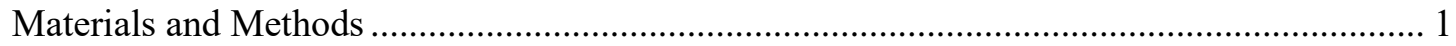

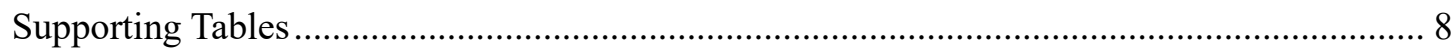

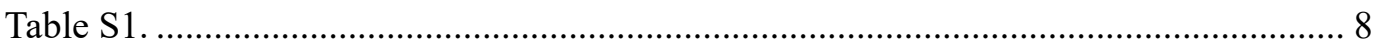

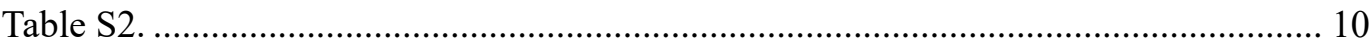

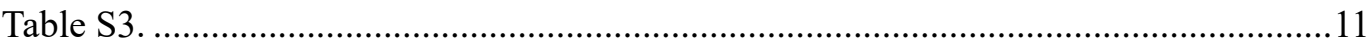

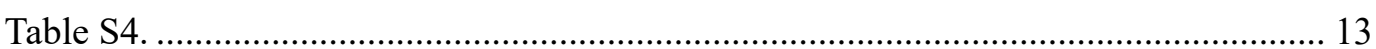

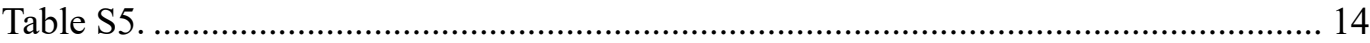

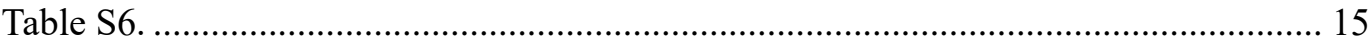

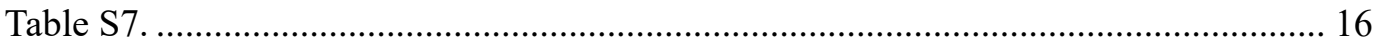

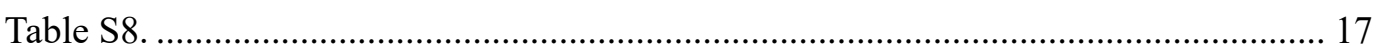

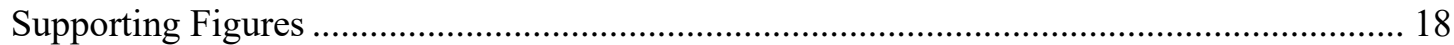

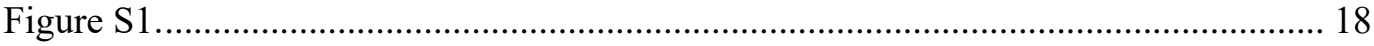

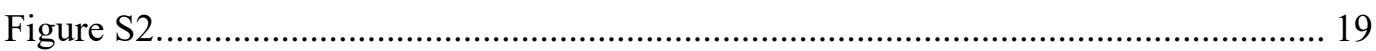

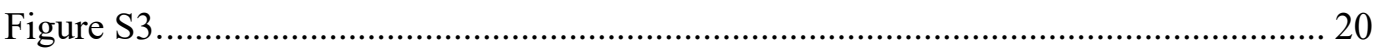

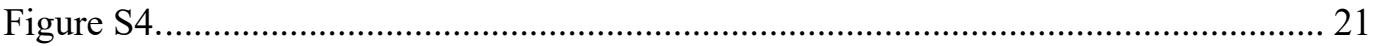

Figure S5

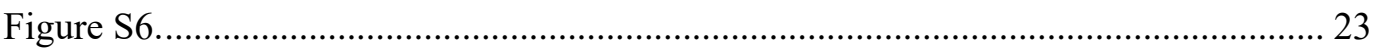

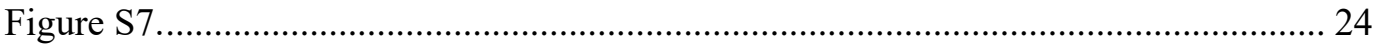

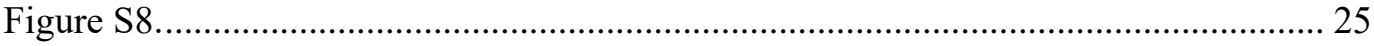

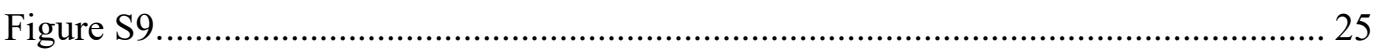

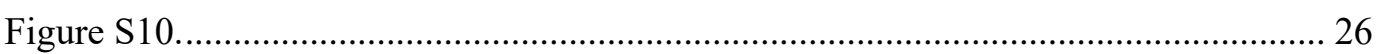

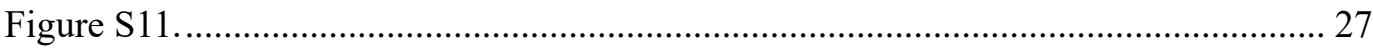

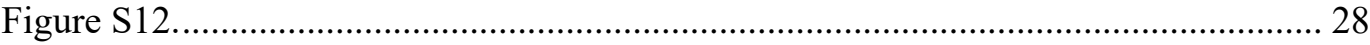

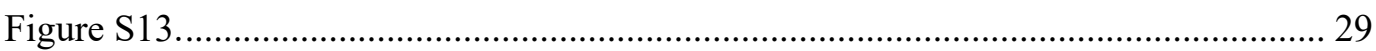

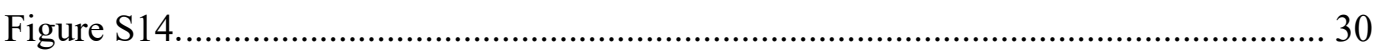

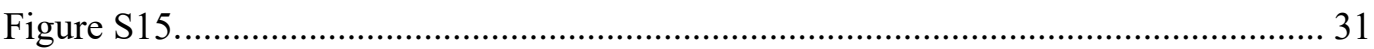




\section{Materials and Methods}

\section{Materials}

DNA primers were ordered by Sangon Biotechnology (Shanghai, China). Reagents used for E. coil and HEK-293T cultivation were purchased from Sangon Biotechnology and Gibco. E. coli strains K-12 MG1655 and DH5a were obtained from MiaolingBio (Wuhan, China). SpCas9 nuclease, Phanta ${ }^{\circledR}$ Max Super-Fidelity DNA Polymerase, Hiscript ${ }^{\circledR}$ III RT SuperMix for qPCR, T7 High Yield Transcription Kit, and iTaq $^{\mathrm{TM}}$ Universal SYBR ${ }^{\circledR}$ Green Supermix were purchased from Vazyme (Nanjing, China). Plasmid DNA was extracted using the Plasmid mini-prep kit (Vazyme), and polymerase chain reaction (PCR) amplicons were purified using the DNA gel extraction kit or PCR product purification kit (Sangon Biotechnology). T4 Ligase and restriction enzymes were purchased from New England Biolabs. Vectors of pET28a, pJ23119, pU6 and pACYC plasmids were obtained from Addgene. Other reagents were obtained from Sangon Biotechnology, unless otherwise indicated.

\section{Plasmid construction}

DH5 $\alpha$ cells were used for general cloning purposes and cultured in Luria-Bertani (LB) media containing appropriate antibiotics. The plasmid pACYC-dCas9 (Streptococcus pyogenes) was constructed by cloning the promoter J23119, RBS and dCas9 into a chloramphenicol-resistant pACYC vector backbone using EcoRI and XhoI sites. For the pJ23119-gRNA-dY and pJ23119gRNA' construction, truncated crRNA/tracrRNA and crRNA $^{\prime} /$ tracrRNA' were cloned into the ampicillin-resistant plasmid under the control of two separate J23119 promotors using EcoRI, BamHI and SalI sites. For the pJ23119-gRNA'-T construction, a separate promoter J23119 and trigger were cloned into pJ23119-gRNA' using SalI and HindIII. For the pJ23119-gRNA'-mCherry construction, a separate promoter J23119, RBS and mCherry were cloned into pJ23119-gRNA' using SalI and XhoI. For the pET28a-gRNA'-lac-operator-mCherry, gRNA' fragment amplified from pJ23119-gRNA', the promoter J23119/J23115/J23103, RBS, the lac operator and the mCherry gene were cloned into the kanamycin-resistant plasmid pET28a. For construction of the pJ23119gRNA'-micF/soxS (--) plasmid, a separate promoter J23115 and single gRNA (sgRNA) for micF or 
soxS were cloned into the corresponding pJ23119-gRNA'. For construction of the pJ23119-gRNA'oxyS (++), a separate promoter J23119 and the oxyS gene were cloned into the corresponding pJ23119-gRNA'. For pU6-gRNA-dY construction, truncated crRNA/tracrRNA were cloned into a kanamycin-resistant plasmid under the control of two separate U6 promotors. For pU6-gRNA'-T construction, crRNA', tracrRNA' and the trigger sequence were cloned into a kanamycin-resistant plasmid under the control of three separate U6 promotors. All these plasmids used in this study were listed in Table S1.

\section{DNA cleavage assay}

For DNA cleavage assays, truncated crRNAs were was chemically synthesized by a K\&A H-8 DNA/RNA Synthesizer using universal CPG as the solid phase support. Truncated tracrRNAs, crRNA', tracrRNA' and the trigger RNA were prepared by T7 in vitro RNA transcriptions using T7 High Yield Transcription Kit according to manufacturer's instructions. All transcribed RNAs were purified by standard phenol-chloroform extraction and ethanol precipitation. The purity of RNA product was confirmed by denatured PAGE.

Cleavage assays were performed using a PCR amplified duplex DNA substrate (dsDNA). To investigate the effect of truncated gRNA on the Cas9 nuclease activity, $100 \mathrm{nM}$ Cas9 protein, 100 nM truncated crRNAs and tracrRNAs, and 100 ng DNA substrate were incubated in the cleavage buffer (20 mM Tris- $\mathrm{HCl}, 100 \mathrm{mM} \mathrm{KCl}, 5 \mathrm{mM} \mathrm{MgCl} 2,1 \mathrm{mM}$ DTT, $0.1 \mathrm{mM}$ EDTA) at $37^{\circ} \mathrm{C}$ for 1 hr. Reaction products were then analyzed by electrophoresis on $1 \%$ agarose gel with ethidium bromide stained, and imaged by Gel Image System (Tanon 2500R).

To illustrate how the ternary gRNA assembly regulated the Cas9 cleavage activity in vitro, $400 \mathrm{nM}$ crRNA $^{\prime}$ and tracrRNA ${ }^{\prime}$ were incubated with or without the trigger $(T)$ or a wrong trigger $\left(\mathrm{T}_{\mathrm{w}}\right)(400$ $\mathrm{nM}$ ) at $37{ }^{\circ} \mathrm{C}$ for $1 \mathrm{hr}$ in the cleavage buffer. The pre-incubated crRNA $/$ tracrRNA'/ Trigger was then mixed with the dsDNA (100 ng) substrate and the Cas9 protein $(100 \mathrm{nM})$, and further incubated at $37^{\circ} \mathrm{C}$ for $1 \mathrm{hr}$. The cleavage products were analyzed as described above. Sequences used in this experiment were listed in Table S2.

\section{Induced transcriptional repression by CRISPRi using artificial trigger strands.}

To compare the regulatory activity between the crRNA/tracrRNA and sgRNA-based CRISPR/Cas9 
function, DH5 $\alpha$ cells were co-transformed with plasmids pACYC-dCas9 (expressing dCas9 for CRISPRi) and pJ23119-gRNA-dY (expressing natural crRNA and tracrRNA) or pJ23119sgRNA (expressing a single gRNA) by heat shock. Single colonies were picked from agar plates and grown in LB media $(10 \mathrm{~g} / \mathrm{L}$ tryptone, $5 \mathrm{~g} / \mathrm{L}$ yeast extract, $10 \mathrm{~g} / \mathrm{L} \mathrm{NaCl})$ supplemented with $100 \mu \mathrm{g} / \mathrm{mL}$ ampicillin, $25 \mu \mathrm{g} / \mathrm{mL}$ chloramphenicol to reach $\mathrm{OD}_{600} \sim 1$ at $37{ }^{\circ} \mathrm{C}$. The cultured bacterial cells were then collected to obtain total RNA.

To study the effect of truncated gRNA on transcriptional repression, DH5 $\alpha$ cells were cotransformed with plasmids pACYC-dCas9 (expressing dCas9 for CRISPRi) and pJ23119-gRNAdY (expressing truncated crRNA and tracrRNA). The transformed cells were cultured under the same conditions to reach $\mathrm{OD}_{600} \sim 1$ as described above. The cultured bacterial cells were then collected to obtain total RNA.

To investigate transcriptional repression controlled by exogenous trigger strands, DH5 $\alpha$ or MG1655 cells were co-transformed with plasmids pACYC-dCas9 (expressing dCas9 for CRISPRi) and pJ23119-gRNA'-T (expressing crRNA', tracrRNA' and Trigger) or pJ23119-gRNA'-mCherry (expressing crRNA', tracrRNA' and mCherry). The transformed cells were cultured under the same conditions to reach $\mathrm{OD}_{600} \sim 1$ as described above. And then the cultured bacterial cells were collected to obtain total RNA.

To study the translation efficiency of mCherry when its mRNA functioned as a trigger to induce the CRISPRi effect, DH5 $\alpha$ were co-transformed with plasmids pACYC-dCas9 and pJ23119gRNA'-mCherry (expressing mCherry, the corresponding or non-binding crRNA'/tracrRNA'). The transformed cells were cultured under the same conditions to reach $\mathrm{OD}_{600} \sim 1$ as described above. The cultured bacterial cells were then collected to determine the fluorescence of mCherry by flow cytometry.

For the IPTG-induced expression of mCherry, pET28a-gRNA'-lac-operator-mCherry (expressing mCherry induced by IPTG) was co-transformed with pACYC-dCas9. The transformed cells were cultured under the same conditions to reach $\mathrm{OD}_{600} \sim 1$ as described above. Expression of mCherry was induced by inoculating fresh LB media with or without the $400 \mu \mathrm{M} \mathrm{IPTG}$ at an initial $\mathrm{OD}_{600} \sim$ 0.03. When reaching $\mathrm{OD}_{600} \sim 1$, the samples were collected to obtain total bacterial RNA. Sequences used in this experiment were listed in Table S3. 


\section{Flow cytometry measurements}

To detect the mCherry fluorescence, the above IPTG-induced samples were collected and washed three times by PBS, followed by suspended in PBS for the flow cytometry analysis on FACS Aria II (BD, USA). For each sample, 10,000 individual cells were detected at the slow rate and the fluorescence intensity was not normalized with the cell density, and analyzed using the Flowjo software.

\section{SRNA and mRNA induced repression}

DH5 $\alpha$ or MG1655 cells were co-transformed with plasmids pACYC-dCas9 (expressing dCas9 for CRISPRi) and pJ23119-gRNA' (expressing crRNA' and tracrRNA') and cultured as described above. The endogenous micF/sgrS/oxyS sRNA and oxyR/soxS/soxR mRNA were utilized to function as a trigger strand to activate the corresponding gRNA'. After reaching $\mathrm{OD}_{600} \sim 1$, the cultured bacterial cells were collected to obtain total RNA. Sequences used in sRNA induced repression experiment were listed in Table S4. Sequences used in mRNA induced repression experiment were listed in Table S5.

\section{Overexpression of oxyS}

To confirm the abundance of native oxyS RNA was too low to induce the effective CRISPRi function, DH5 $\alpha$ cells were co-transformed with plasmids pACYC-dCas9 (expressing dCas9 for CRISPRi) and pJ23119-gRNA'-oxyS (++) (expressing crRNA'/tracrRNA' and oxyS). The transformed cells were cultured under the same conditions to reach $\mathrm{OD}_{600} \sim 1$ as described above. Then the cultured bacterial cells were collected to obtain total RNA. Sequences used in this experiment were listed in Table S4.

\section{Knockdown of Micf or SoxS induced gene regulation}

DH5 $\alpha$ cells were co-transformed with plasmids pACYC-dCas9 (expressing dCas9 for CRISPRi) and pJ23119-gRNA'-micF (--) (expressing crRNA'/tracrRNA' to target the independent galA gene and micF-sgRNA for knockdown of micF) or pJ23119-gRNA'-soxS (--) (expressing crRNA'/tracrRNA' to target the independent galA gene and soxS-sgRNA for knockdown of soxS). Same vectors with the incorrect crRNA'/tracrRNA' that could not be linked by micF or soxS were 
selected as controls. The transformed cells were cultured under the same conditions to reach $\mathrm{OD}_{600}$ $\sim 1$ as described above. Then the cultured bacterial cells were collected to obtain total RNA. Sequences related to the micF knockdown experiments were listed in Table S4. Sequences related to the SoxS knockdown experiments were listed in Table S5.

\section{qPCR for determination of RNA expression levels in E. coil}

To obtain bacterial RNA, cell samples were pelleted by centrifugation at 3,000 g for $5 \mathrm{~min}$, and washed twice with PBS. The total RNA was extracted using bacterial total RNA Rapid extraction kits (Sangon Biotechnology) according to the manufacturer's instructions. The extracted total RNA was quantified by the UV-Vis spectrophotometry and the value of OD260/280 was between 1.8 and 2.0 .

For cDNA synthesis, the total RNA was reverse transcribed using the cDNA Synthesis Kit (Vazyme). Briefly, $1 \mu \mathrm{g}$ total RNA was mixed with the RT SuperMix (containing dNTP, reaction buffer, reverse transcriptase, RNase inhibitor and random primers) in a total reaction volume of $20 \mu \mathrm{L}$. Reverse transcriptions (RT) were conducted at $37^{\circ} \mathrm{C}$ for $15 \mathrm{~min}$ to obtain cDNA, followed by inactivation of the reverse transcriptase at $85^{\circ} \mathrm{C}$ for $15 \mathrm{~s}$. All RT reactions were performed on the T100 Thermal cycler (BIO-RAD). For cDNA synthesis of mCherry and overexpressed-oxyS, total RNA was pretreated with DNase I (Vazyme) to remove plasmid DNA at $37^{\circ} \mathrm{C}$ for $30 \mathrm{~min}$ and then at $95^{\circ} \mathrm{C}$ for 5 min to deactivate DNase I before reverse transcriptions.

The qPCR experiments were carried out in the reaction mixture containing SYBR ${ }^{\circledR}$ Green Supermix (Vazyme), $0.2 \mu \mathrm{M}$ the forward primer, $0.2 \mu \mathrm{M}$ the reverse primer and the diluted cDNA using the CFX96 Real-Time system (BIO-RAD). Reference genes (rssA and 16S RNA) were used to normalize the sRNA and mRNA expression levels, respectively. The thermocycling conditions for mRNA were: $95{ }^{\circ} \mathrm{C}$ for $10 \mathrm{~min}$ followed by 40 cycles of $95{ }^{\circ} \mathrm{C}$ for $15 \mathrm{~s}$ and $62{ }^{\circ} \mathrm{C}$ for $1 \mathrm{~min}$. The thermocycling conditions for sRNA were: $95^{\circ} \mathrm{C}$ for 10 min followed by 40 cycles of $95{ }^{\circ} \mathrm{C}$ for 15 $\mathrm{s}$ and $58{ }^{\circ} \mathrm{C}$ for $1 \mathrm{~min}$. The purity of the PCR product was validated by the electrophoresis gel and the melting profile. The Cycle-Threshold $(\mathrm{Ct})$ values were determined by the default settings of the software. The qPCR results were only determined as effective data when the $\mathrm{Ct}$ values were less than 35. Reference genes were used to normalize expression levels using the $2^{-\Delta \Delta \mathrm{CT}}$ method. Three technical replicates were performed for each PCR reaction. Primers used in this study were listed in 
Table S8.

\section{Growth profiling experiments}

E. coli K-12 MG1655 was used for growth profiling experiments. E. coli K-12 MG1655 were cotransformed with plasmids pACYC-dCas9 (expressing dCas9 for CRISPRi) and pJ23119-gRNA'-T (crRNA', tracrRNA' and Trigger) or pJ23119-gRNA' (crRNA' and tracrRNA'). The growth profiling experiments ${ }^{1}$ were performed in the M9 medium with $0.4 \%(\mathrm{w} / \mathrm{v})$ glucose and $0.04 \%(\mathrm{w} / \mathrm{v})$ yeast extract (M9G0.4YE0.04) containing appropriate antibiotics. In details, single colonies were inoculated into M9G0.4YE0.04 with appropriate antibiotics for overnight growth at $37^{\circ} \mathrm{C}$ and 250 rpm. The overnight culture was diluted by 100-fold into fresh M9G0.4YE0.04 media with corresponding antibiotics. For each colony, three $150 \mu \mathrm{L}$ parallel cultures were prepared and transferred into 96-well microtiter plates for growth profiling. Plates were cultivated in a microplate reader (BioTek Gene5, USA) at $37^{\circ} \mathrm{C}$ with the medium shaking intensity, and the optical density (OD) at $600 \mathrm{~nm}$ for each well was recorded at the intervals of $10 \mathrm{~min}$. The growth curves were presented from three independent colonies with the data of each colony obtained from three parallel cultures. Sequences used in this experiment were listed in Table S6.

To precisely quantify the growth rate of E. coil, an asymmetric five-parameter logistic equation was utilized to fit the growth curves of E. coil:

Denominator $=\left(1+\left(2^{\wedge}(1 / \mathrm{S})-1\right) \times\left(\left(\mathrm{t}_{1 / 2} / \mathrm{X}\right)^{\wedge} \text { Hillslope }\right)\right)^{\wedge} \mathrm{S}$

Numerator $=$ Top - Bottom

$\mathrm{Y}=$ Bottom $+($ Numerator/Denominator $)$

where Bottom is the point of minimum $\mathrm{OD}_{600}$, Top is the point of maximum $\mathrm{OD}_{600}, \mathrm{t}_{1 / 2}$ is the time reaching to the half of maximum $\mathrm{OD}_{600}$, Hillslope is the unitless slope factor, and $\mathrm{S}$ is the unitless symmetry parameter.

\section{Transcriptional regulation in HEK293T cells}

293T-dCas9-VPR cells were prepared and cultured as described in our previous study². To investigate the effect of truncated gRNA on transcriptional regulation of the ASCL1 gene, 250,000 293T-dCas9-VPR cells were seeded in 6-well plates, and immediately transfected with $2000 \mathrm{ng}$ of pU6-gRNA-dY encoding truncated crRNA and tracrRNA using $5.5 \mu \mathrm{L}$ Lipofectamine 3000 and 4 
$\mu \mathrm{L}$ P3000 (Invitrogen). Cells were then collected for determination of RNA expression levels after one-day culture.

To investigate transcriptional activation controlled by exogenous trigger strands, 293T-dCas9-VPR cells were transfected with pU6-gRNA'-T (expressing crRNA', tracrRNA' and Trigger) following the same method. And after one-day culture, cells were collected for determination of RNA expressions. Sequences used in this experiment were listed in Table S7.

Total RNA from 293T cells was isolated using trizol (life technology), chloroform (Sigma), isopropanol and ethyl alcohol, and quantified by the UV-Vis spectrophotometry and the value of OD 260/280 was between 1.8 and 2.0. For the reverse transcription of mRNA, $1 \mu \mathrm{g}$ total RNA was used to generate cDNA through the poly(dT) primer using cDNA Synthesis Kit (Vazyme) according to the manufacturer's instructions as described above.

The qPCR experiments were carried out in the reaction mixture containing SYBR ${ }^{\circledR}$ Green Supermix (Vazyme), $0.2 \mu \mathrm{M}$ forward primer, $0.2 \mu \mathrm{M}$ reverse primer and diluted cDNA using the CFX96 RealTime system (BIO-RAD) as described above. The ASCL1 mRNA levels were normalized using the expression level of GAPDH as the control. 


\section{Supporting Tables}

Table S1. Major plasmid constructs in this work.

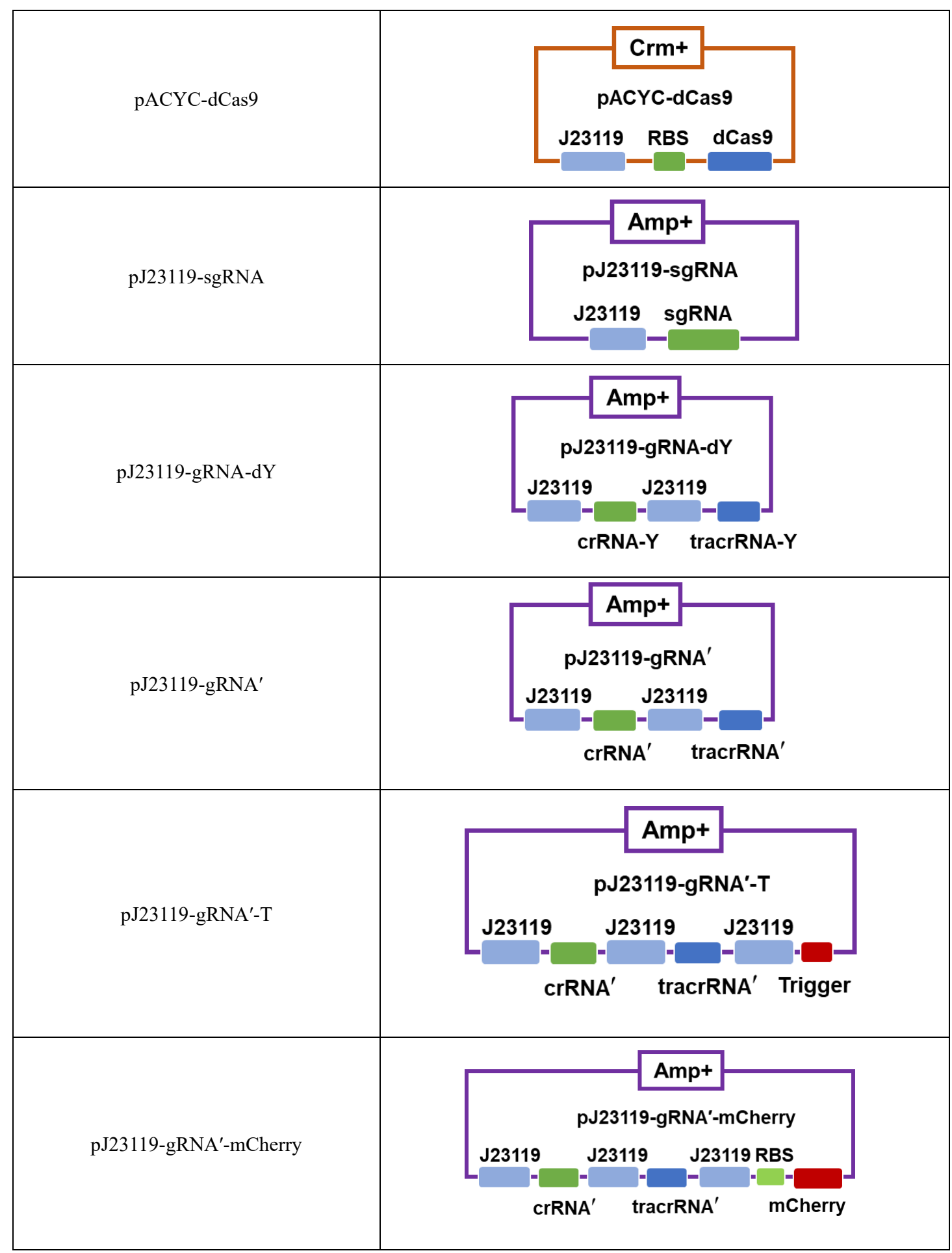




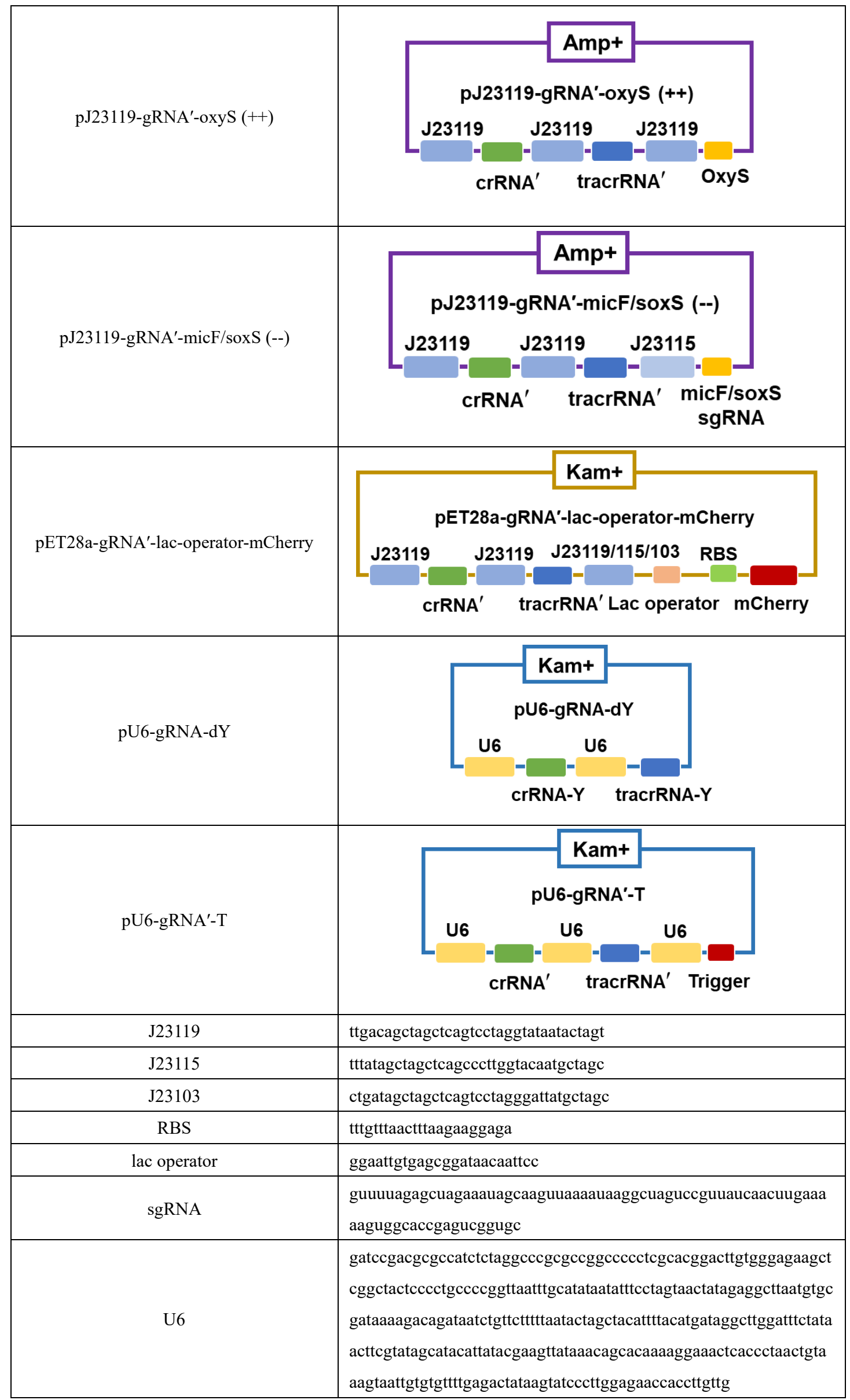


Table S2. Truncated crRNA/tracrRNA and crRNA'/tracrRNA' sequences used in Figure 1 and Figure S1.

\begin{tabular}{|c|c|}
\hline & RNA sequence (5'-3') \\
\hline crRNA-14 & 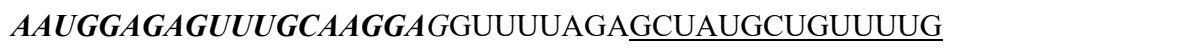 \\
\hline crRNA-10 & 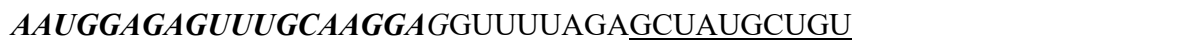 \\
\hline crRNA-6 & AAUGGAGAGUUUGCAAGGAGGUUUAGAGCUAUG \\
\hline crRNA-4 & 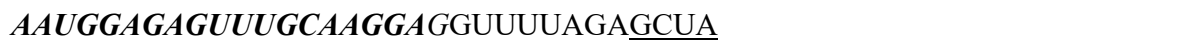 \\
\hline crRNA-3 & 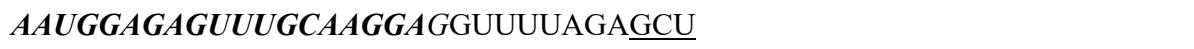 \\
\hline crRNA-2 & 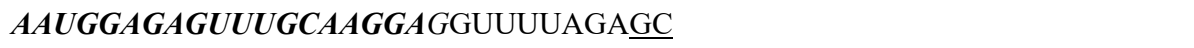 \\
\hline crRNA-1 & 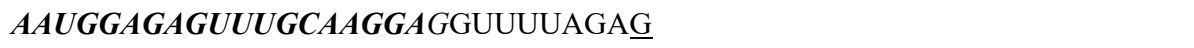 \\
\hline crRNA-0 & AAUGGAGAGUUUGCAAGGAGGUUUUAGA \\
\hline tracrRNA-14 & $\begin{array}{l}\text { CAAAACAGCAUAGCAAGUUAAAAUAAGGCUAGUCCGUUAUCAACUUGAAAAAG } \\
\text { UGGCACCGAGUCGGUGCU }\end{array}$ \\
\hline tracrRNA-10 & $\begin{array}{l}\text { ACAGCAUAGCAAGUUAAAAUAAGGCUAGUCCGUUAUCAACUUGAAAAAGUGGC } \\
\text { ACCGAGUCGGUGCU }\end{array}$ \\
\hline tracrRNA-6 & $\begin{array}{l}\text { CAUAGCAAGUUAAAAUAAGGCUAGUCCGUUAUCAACUUGAAAAAGUGGCACCG } \\
\text { AGUCGGUGCU }\end{array}$ \\
\hline tracrRNA-4 & $\begin{array}{l}\text { UAGCAAGUUAAAAUAAGGCUAGUCCGUUAUCAACUUGAAAAAGUGGCACCGAG } \\
\text { UCGGUGCU }\end{array}$ \\
\hline tracrRNA-3 & $\begin{array}{l}\text { AGCAAGUUAAAAUAAGGCUAGUCCGUUAUCAACUUGAAAAAGUGGCACCGAGU } \\
\text { CGGUGCU }\end{array}$ \\
\hline tracrRNA-2 & $\begin{array}{l}\text { GCAAGUUAAAAUAAGGCUAGUCCGUUAUCAACUUGAAAAAGUGGCACCGAGUC } \\
\text { GGUGCU }\end{array}$ \\
\hline tracrRNA-1 & $\begin{array}{l}\text { CAAGUUAAAAUAAGGCUAGUCCGUUAUCAACUUGAAAAAGUGGCACCGAGUCG } \\
\text { GUGCU }\end{array}$ \\
\hline tracrRNA-0 & $\begin{array}{l}\text { AAGUUAAAAUAAGGCUAGUCCGUUAUCAACUUGAAAAAGUGGCACCGAGUCGG } \\
\text { UGCU }\end{array}$ \\
\hline crRNA' & $\boldsymbol{A} \boldsymbol{A} \boldsymbol{U} \boldsymbol{G} \boldsymbol{G} \boldsymbol{A} \boldsymbol{G} \boldsymbol{A} \boldsymbol{G} \boldsymbol{U} \boldsymbol{U} \boldsymbol{U} \boldsymbol{G} \boldsymbol{C A} \boldsymbol{A} \boldsymbol{G} \boldsymbol{G} \boldsymbol{A} \boldsymbol{G G U U U U A G A A G A U U G A A G C C A U A C C A A A C G ~}$ \\
\hline tracrRNA' & $\begin{array}{l}\text { UUGAAGCAAGCCAACCGGACCAAGUUAAAAUAAGGCUAGUCCGUUAUCAACUU } \\
\text { GAAAAAGUGGCACCGAGUCGGUGCU }\end{array}$ \\
\hline Trigger $(\mathrm{T})$ & CGUUUGGUAUGGCUUCAAUCUGGUCCGGUUGGCUUGCUUCAA \\
\hline $\mathrm{T}_{\mathrm{w}}$ & GGCAACAACGUUGCGCAAACUAUAAGUCCCACUCGGAUCGAG \\
\hline
\end{tabular}


Table S3. RNA sequences used in Figure 2 and Figure S3, Figure S4, Figure S6.

\begin{tabular}{|c|c|}
\hline & RNA sequence (5'-3') \\
\hline \multicolumn{2}{|l|}{ galA-gRNA-d14 } \\
\hline crRNA-14 & 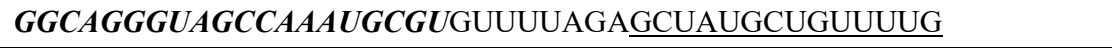 \\
\hline tracrRNA14 & $\begin{array}{l}\text { CAAAACAGCAUAGCAAGUUAAAAUAAGGCUAGUCCGUUAUCAACUUGAA } \\
\text { AAAGUGGCACCGAGUCGGUGCU }\end{array}$ \\
\hline \multicolumn{2}{|l|}{ galA-gRNA-d10 } \\
\hline crRNA-10 & 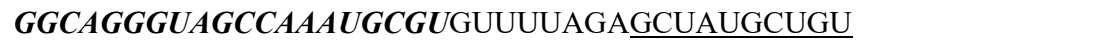 \\
\hline tracrRNA10 & $\begin{array}{l}\text { ACAGCAUAGCAAGUUAAAAUAAGGCUAGUCCGUUAUCAACUUGAAAAAG } \\
\text { UGGCACCGAGUCGGUGCU }\end{array}$ \\
\hline \multicolumn{2}{|l|}{ galA-gRNA-d6 } \\
\hline crRNA-6 & 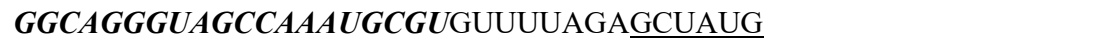 \\
\hline tracrRNA-6 & $\begin{array}{l}\text { CAUAGCAAGUUAAAAUAAGGCUAGUCCGUUAUCAACUUGAAAAAGUGGC } \\
\text { ACCGAGUCGGUGCU }\end{array}$ \\
\hline \multicolumn{2}{|l|}{ galA-gRNA-d4 } \\
\hline crRNA-4 & GGCAGGGUAGCCAAAUGCGUGUUUUAGAGCUA \\
\hline tracrRNA-4 & $\begin{array}{l}\text { UAGCAAGUUAAAAUAAGGCUAGUCCGUUAUCAACUUGAAAAAGUGGCAC } \\
\text { CGAGUCGGUGCU }\end{array}$ \\
\hline \multicolumn{2}{|l|}{ galA-gRNA-d3 } \\
\hline crRNA-3 & GGCAGGGUAGCCAAAUGCGUGUUUUAGAGCU \\
\hline tracrRNA-3 & $\begin{array}{l}\text { AGCAAGUUAAAAUAAGGCUAGUCCGUUAUCAACUUGAAAAAGUGGCACC } \\
\text { GAGUCGGUGCU }\end{array}$ \\
\hline \multicolumn{2}{|l|}{ galA-gRNA-d2 } \\
\hline crRNA-2 & GGCAGGGUAGCCAAAUGCGUGUUUUAGAGC \\
\hline tracrRNA-2 & $\begin{array}{l}\text { GCAAGUUAAAAUAAGGCUAGUCCGUUAUCAACUUGAAAAAGUGGCACCG } \\
\text { AGUCGGUGCU }\end{array}$ \\
\hline \multicolumn{2}{|l|}{ galA-gRNA-d1 } \\
\hline crRNA-1 & 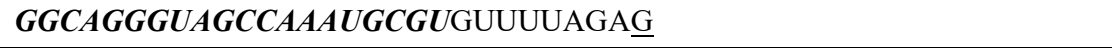 \\
\hline tracrRNA-1 & $\begin{array}{l}\text { CAAGUUAAAAUAAGGCUAGUCCGUUAUCAACUUGAAAAAGUGGCACCGA } \\
\text { GUCGGUGCU }\end{array}$ \\
\hline \multicolumn{2}{|l|}{ galA-gRNA-d0 } \\
\hline crRNA-0 & 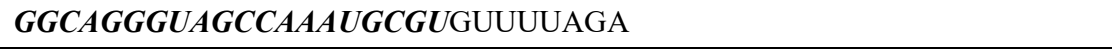 \\
\hline tracrRNA-0 & $\begin{array}{l}\text { AAGUUAAAAUAAGGCUAGUCCGUUAUCAACUUGAAAAAGUGGCACCGAG } \\
\text { UCGGUGCU }\end{array}$ \\
\hline \multicolumn{2}{|l|}{ galA-gRNA'-9 bp } \\
\hline crRNA'-9 & 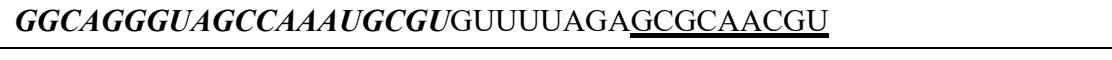 \\
\hline tracrRNA'-9 & $\begin{array}{l}\text { UUAUAGUUUAAGUUAAAAUAAGGCUAGUCCGUUAUCAACUUGAAAAAGU } \\
\text { GGCACCGAGUCGGUGCU }\end{array}$ \\
\hline Trigger & ACGUUGCGCAAACUAUAA \\
\hline \multicolumn{2}{|l|}{ galA-gRNA'-12bp } \\
\hline crRNA'-12 & 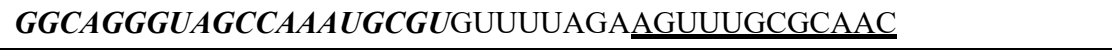 \\
\hline tracrRNA'-12 & $\underline{\text { AGUGGGACUUAUAAGUUAAAAUAAGGCUAGUCCGUUAUCAACUUGAAAA }}$ \\
\hline
\end{tabular}




\begin{tabular}{|c|c|}
\hline & AGUGGCACCGAGUCGGUGCU \\
\hline Trigger & GUUGCGCAAACUAUAAGUCCCACU \\
\hline \multicolumn{2}{|c|}{ galA-gRNA'-15bp } \\
\hline crRNA'-15 & GGCAGGGUAGCCAAAUGCGUGUUUUAGAAGUUUGCGCAACGUU \\
\hline tracrRNA'-15 & $\begin{array}{l}\text { CCGAGUGGGACUUAUAAGUUAAAAUAAGGCUAGUCCGUUAUCAACUUGA } \\
\text { AAAAGUGGCACCGAGUCGGUGCU }\end{array}$ \\
\hline Trigger & AACGUUGCGCAAACUAUAAGUCCCACUCGG \\
\hline \multicolumn{2}{|c|}{ galA-gRNA'-18bp } \\
\hline crRNA'-18 & 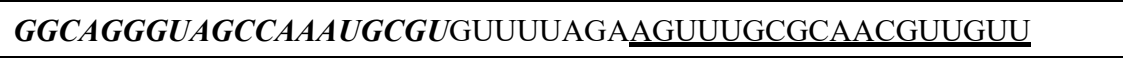 \\
\hline tracrRNA'-18 & $\begin{array}{l}\text { GAUCCGAGUGGGACUUAUAAGUUAAAAUAAGGCUAGUCCGUUAUCAACU } \\
\text { UGAAAAAGUGGCACCGAGUCGGUGCU }\end{array}$ \\
\hline Trigger & AACAACGUUGCGCAAACUAUAAGUCCCACUCGGAUC \\
\hline \multicolumn{2}{|c|}{ galA-gRNA'-21bp } \\
\hline crRNA'-21 & 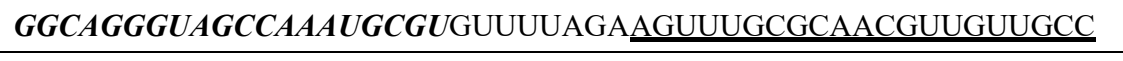 \\
\hline tracrRNA'-21 & $\begin{array}{l}\text { CUCGAUCCGAGUGGGACUUAUAAGUUAAAAUAAGGCUAGUCCGUUAUCA } \\
\text { ACUUGAAAAAGUGGCACCGAGUCGGUGCU }\end{array}$ \\
\hline Trigger $(\mathrm{T})$ & GGCAACAACGUUGCGCAAACUAUAAGUCCCACUCGGAUCGAGC \\
\hline $\mathrm{T}_{\mathrm{w}}$ & UUACACUGAUGGGGCGAUCGGUGCCCAUCAGUGUA \\
\hline \multicolumn{2}{|l|}{ Strand 1} \\
\hline crRNA' & 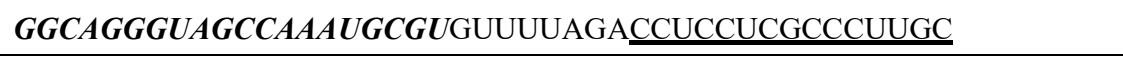 \\
\hline tracrRNA' & $\begin{array}{l}\text { UGAUGGCCAUGUUAUAAGUUAAAAUAAGGCUAGUCCGUUAUCAACUUGA } \\
\text { AAAAGUGGCACCGAGUCGGUGCU }\end{array}$ \\
\hline Trigger & GCAAGGGCGAGGAGGAUAACAUGGCCAUCA \\
\hline \multicolumn{2}{|l|}{ Strand 2} \\
\hline crRNA' & GGCAGGGUAGCCAAAUGCGUGUUUUAGACUCGAUCUCGAACUC \\
\hline tracrRNA' & $\begin{array}{l}\text { GCCCUCGCCCUCGCCAAGUUAAAAUAAGGCUAGUCCGUUAUCAACUUGAA } \\
\text { AAAGUGGCACCGAGUCGGUGCU }\end{array}$ \\
\hline Trigger & GAGUUCGAGAUCGAGGGCGAGGGCGAGGGC \\
\hline \multicolumn{2}{|l|}{ Strand 3} \\
\hline crRNA' & 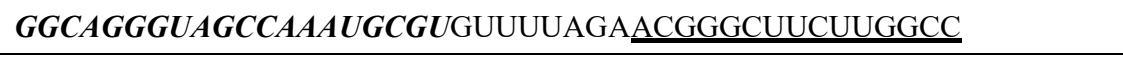 \\
\hline tracrRNA' & $\begin{array}{l}\text { GCGCCGGGCAGCUGCAAGUUAAAAUAAGGCUAGUCCGUUAUCAACUUGA } \\
\text { AAAAGUGGCACCGAGUCGGUGCU }\end{array}$ \\
\hline Trigger & GGCCAAGAAGCCCGUGCAGCUGCCCGGCGC \\
\hline mCherry & 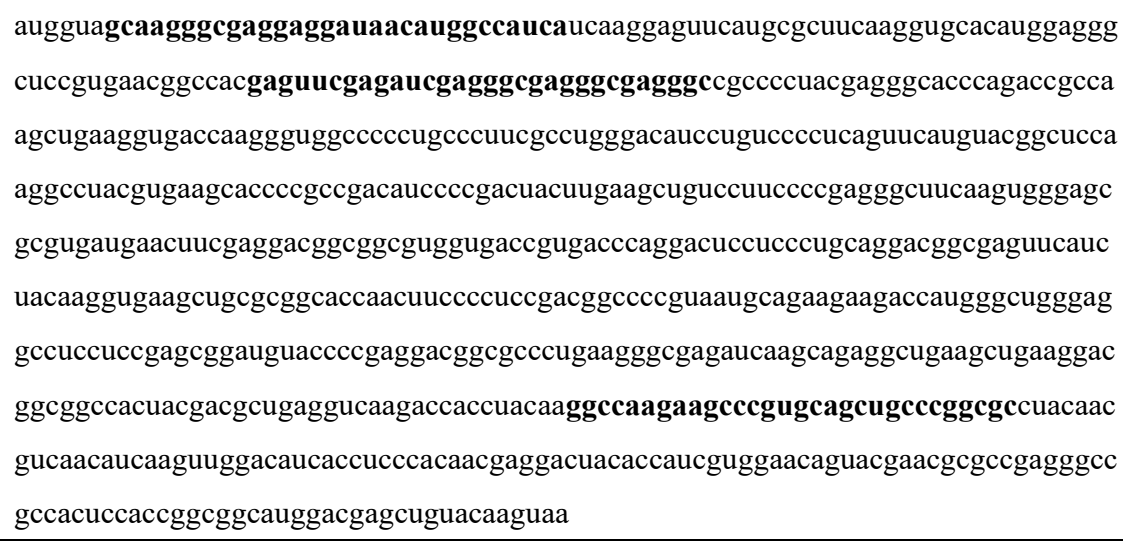 \\
\hline
\end{tabular}




\begin{tabular}{|c|c|}
\hline lacZ-crRNA14 & UUGGGAAGGGCGAUCGGUGCGUUUUAGAGCUAUGCUGUUUUG \\
\hline lacZ-crRNA'-15 & 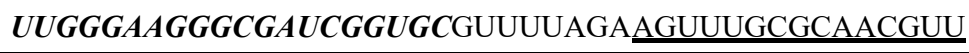 \\
\hline Null-crRNA & GUUUUAGAGCUAUGCUGUUUUG \\
\hline
\end{tabular}

Table S4. RNA sequences used in Figure 3 and Figure S10, Figure S11.

\begin{tabular}{|c|c|}
\hline & RNA sequence (5'-3') \\
\hline $\mathrm{micF}$ & $\begin{array}{l}\text { gcuaucaucauuaacuunauuuauuaccgucauucauuucugaaugucuguuuaccccuauuucaaccggau } \\
\text { gccucgcauccgguuuuuuuu }\end{array}$ \\
\hline galA-crRNA' & 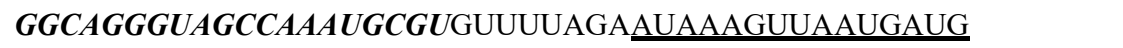 \\
\hline lacZ-crRNA' & $\boldsymbol{U} \boldsymbol{U} \boldsymbol{G} \boldsymbol{G} \boldsymbol{G A} \boldsymbol{A} \boldsymbol{G} \boldsymbol{G} \boldsymbol{G} \boldsymbol{C} \boldsymbol{G} \boldsymbol{A} \boldsymbol{U} \boldsymbol{C} \boldsymbol{G} \boldsymbol{G} \boldsymbol{U} \boldsymbol{G} \boldsymbol{C G U U U U A G A} \underline{\mathrm{AUAAAGUUAAUGAUG}}$ \\
\hline tracrRNA' & $\begin{array}{l}\text { GAAUGACGGUAAUAAAAGUUAAAAUAAGGCUAGUCCGUUAUCAACUUGA } \\
\text { AAAAGUGGCACCGAGUCGGUGCU }\end{array}$ \\
\hline $\operatorname{sgrS}$ & $\begin{array}{l}\text { gaugaagcaagggggugccccaugcgucaguuuuaucagcacuauuuuaccgcgacagcgaaguugugcugguu } \\
\text { gcguugguuaagcgucccacaacgauuaaccaugcuugaaggacugaugcagugggaugaccgcaauucugaaag } \\
\text { uugacuugccugcaucaugugugacugaguauugguguaaaaucaccegccagcagauuauaccugcugguu } \\
\text { uuuuuu }\end{array}$ \\
\hline galA-crRNA' & $\boldsymbol{G} \boldsymbol{G C} \boldsymbol{A} \boldsymbol{G} \boldsymbol{G} \boldsymbol{G} \boldsymbol{U} \boldsymbol{A} \boldsymbol{G} \boldsymbol{C C} \boldsymbol{A} \boldsymbol{A} \boldsymbol{A} \boldsymbol{U} \boldsymbol{G} \boldsymbol{C G} \boldsymbol{U} G U U U U \mathrm{AGAGUCACACAUGAUGCA}$ \\
\hline tracrRNA' & $\begin{array}{l}\text { UUACACCAAUACUCAAAGUUAAAAUAAGGCUAGUCCGUUAUCAACUUGA } \\
\text { AAAAGUGGCACCGAGUCGGUGCU }\end{array}$ \\
\hline oxyS & $\begin{array}{l}\text { gaaacggagcggcaccucuuuuaacccuugaagucacugcccguuucgagaguuucucaacucgaauaacuaaa } \\
\text { gccaacgugaacuuuugcggaucuccaggauccgcu }\end{array}$ \\
\hline galA-crRNA' & 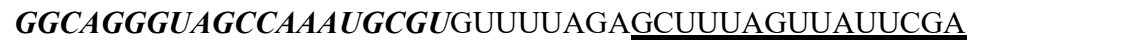 \\
\hline tracrRNA' & $\begin{array}{l}\text { CAAAAGUUCACGUUGAAGUUAAAAUAAGGCUAGUCCGUUAUCAACUUGA } \\
\text { AAAAGUGGCACCGAGUCGGUGCU }\end{array}$ \\
\hline Control & \\
\hline galA-crRNA' & GGCAGGGUAGCCAAAUGCGUGUUUUAGACCUCCUCGCCCUUGC \\
\hline tracrRNA' & $\begin{array}{l}\text { UGAUGGCCAUGUUAUAAGUUAAAAUAAGGCUAGUCCGUUAUCAACUUGA } \\
\text { AAAAGUGGCACCGAGUCGGUGCU }\end{array}$ \\
\hline micF-sgRNA & 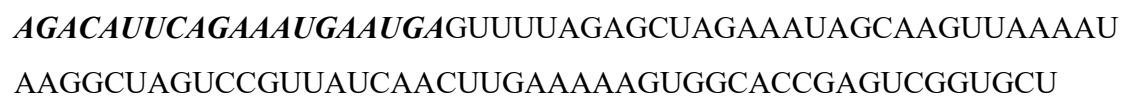 \\
\hline Null-sgRNA & $\begin{array}{l}\text { GUUUUAGAGCUAGAAAUAGCAAGUUAAAAUAAGGCUAGUCCGUUAUCAA } \\
\text { CUUGAAAAAGUGGCACCGAGUCGGUGCU }\end{array}$ \\
\hline
\end{tabular}


Table S5. RNA sequences used in Figure 4 and Figure S12.

\begin{tabular}{|c|c|}
\hline & RNA sequence (5'-3') \\
\hline oxyR & $\begin{array}{l}\text { augaauauucgugaucuugaguaccugguggcauuggcugaacaccgccauuuucggcgugcggcagauucc } \\
\text { ugccacguuagccagccgacgcuuagcgggcaauucguaagcuggaagaugagcugggcgugauguugcugga } \\
\text { gcggaccagccguaaaguguuguucacccaggcgggaaugcugcugguggaucaggcgeguaccgugcugcgu } \\
\text { gaggugaaaguccuuaaagagauggcaagccagcagggcgagacgauguccggaccgcugcacauugguuugau } \\
\text { ucccacaguuggaccguaccugcuaccgcauauuaucccuaugcugcaccagaccuuuccaaagcuggaaaugua } \\
\text { ucugcaugaagcacagacccaccaguuacuggcgcaacuggacagcggcaaacucgauugcgugauccucgcgcu } \\
\text { ggugaaagagagcgaagcauucauugaagugccguuguuugaugagccaauguugcuggcuaucuaugaagauc } \\
\text { acccgugggcgaaccgcgaaugcguaccgauggccgaucuggcaggggaaaacugcugaugcuggaagauggu } \\
\text { cacuguuugcgcgaucaggcaauggguuucuguuuugaagccggggcggaugaagauacacacuuccgcgcgac } \\
\text { cagccuggaaacucugcgcaacaugguggcggcagguagcgggaucacuuuacugccagcgcuggcugugccgc } \\
\text { cggagcgcaaacgcgaugggguuguuuaucugccgugcauuaagccggaaccacgccgcacuauuggccugguu } \\
\text { uaucguccuggcucaccgcugcgcagccgcuaugagcagcuggcagaggccauccgcgcaagaauggauggcca } \\
\text { uuucgauaaaguuuuaaaacaggcgguuuaa }\end{array}$ \\
\hline \multicolumn{2}{|l|}{ Site 1} \\
\hline crRNA' & 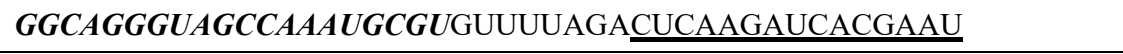 \\
\hline tracrRNA' & $\begin{array}{l}\text { CAATGCCACCAGGTAAAGUUAAAAUAAGGCUAGUCCGUUAUCAACUUGAA } \\
\text { AAAGUGGCACCGAGUCGGUGCU }\end{array}$ \\
\hline \multicolumn{2}{|l|}{ Site 2} \\
\hline crRNA' & GGCAGGGUAGCCAAAUGCGUGUUUUAGAUGAUCCACCAGCAGC \\
\hline tracrRNA' & $\begin{array}{l}\text { AGCACGGUACGCGCCAAGUUAAAAUAAGGCUAGUCCGUUAUCAACUUGA } \\
\text { AAAAGUGGCACCGAGUCGGUGCU }\end{array}$ \\
\hline \multicolumn{2}{|r|}{ I, } \\
\hline crRNA' $^{\prime}$ & 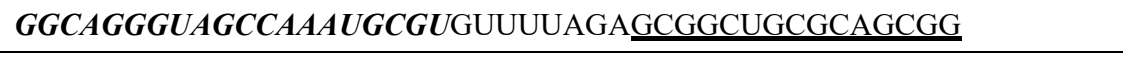 \\
\hline tracrRNA' & $\begin{array}{l}\text { UGCCAGCUGCUCAUAAAGUUAAAAUAAGGCUAGUCCGUUAUCAACUUGA } \\
\text { AAAAGUGGCACCGAGUCGGUGCU }\end{array}$ \\
\hline $\operatorname{sox} R$ & $\begin{array}{l}\text { auggaaaagaaauuaccegcauuaaagcgcugcuaacccceggcgaaguggcgaaacgcagcgguguggcgg } \\
\text { uaucggcgcugcauuucuaugaaaguaaaggguugauuaccaguauccguaacagcggcaaucagcggcgauaua } \\
\text { aacgugauguguugcgauauguugcaauuaucaaaauugcucagcguauuggcauuccgcuggcgaccauugg } \\
\text { ugaagcguuuggcguguugcccgaagggcauacguuaagugcgaaagaguggaaacagcuuucgucccaauggc } \\
\text { gagaagaguuggaucggcgcauucauaccuuaguggcgcugcgugacgaacuggacggauguauugguuguggc } \\
\text { ugccuuucgcgcagugauugcceguugcguaaccegggcgaccgcuuaggagaagaagguaccggcgcacgcu } \\
\text { ugcuggaagaugaacaaaacuaa }\end{array}$ \\
\hline \multicolumn{2}{|l|}{ Site 1} \\
\hline crRNA' $^{\prime}$ & GGCAGGGUAGCCAAAUGCGUGUUUUAGAGCGGGGUAAUUUCUU \\
\hline tracrRNA' & $\begin{array}{l}\text { UAGCAGCGCUUUAAUAAGUUAAAAUAAGGCUAGUCCGUUAUCAACUUGA } \\
\text { AAAAGUGGCACCGAGUCGGUGCU }\end{array}$ \\
\hline \multicolumn{2}{|l|}{ Site 2} \\
\hline crRNA' & GGCAGGGUAGCCAAAUGCGUGUUUUAGACGCCAGCGGAAUGCC \\
\hline tracrRNA' & $\begin{array}{l}\text { CGCUUCACCAAUGGUAAGUUAAAAUAAGGCUAGUCCGUUAUCAACUUGA } \\
\text { AAAAGUGGCACCGAGUCGGUGCU }\end{array}$ \\
\hline
\end{tabular}




\begin{tabular}{|c|c|}
\hline Site 3 & \\
\hline crRNA' & 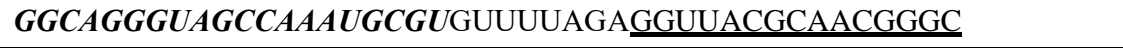 \\
\hline tracrRNA' & $\begin{array}{l}\text { CUAAGCGGUCGCCCGAAGUUAAAAUAAGGCUAGUCCGUUAUCAACUUGA } \\
\text { AAAAGUGGCACCGAGUCGGUGCU }\end{array}$ \\
\hline $\operatorname{sox} S$ & $\begin{array}{l}\text { augucccaucagaaauuauucaggaucuuaucgcauggauugacgagcauauugaccagccgcuuaacauug } \\
\text { auguagucgcaaaaaaucaggcuauucaaagugguacuugcaacgaauguuccgcacggugacgcaucagacgc } \\
\text { uuggcgauuacauucgccaacgccgccuguuacuggccgecguugaguugcgcaccaccgagcguccgauuuu } \\
\text { ugauaucgcaauggaccuggguuaugucucgcagcagaccuucuccegcguuuuccgucggcaguuugaucge } \\
\text { acucccagcgauuaucgccaccgccuguaa }\end{array}$ \\
\hline \multicolumn{2}{|l|}{ Site 1} \\
\hline crRNA' & 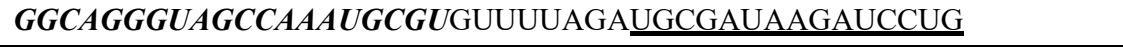 \\
\hline tracrRNA' & $\begin{array}{l}\text { AUGCUCGUCAAUCCAAAGUUAAAAUAAGGCUAGUCCGUUAUCAACUUGA } \\
\text { AAAAGUGGCACCGAGUCGGUGCU }\end{array}$ \\
\hline \multicolumn{2}{|l|}{ Site 2} \\
\hline crRNA' & GGCAGGGUAGCCAAAUGCGUGUUUUAGACAGGCGGCGUUGGCG \\
\hline tracrRNA' & $\begin{array}{l}\text { AACGGCGGCCAGUAAAAGUUAAAAUAAGGCUAGUCCGUUAUCAACUUGA } \\
\text { AAAAGUGGCACCGAGUCGGUGCU }\end{array}$ \\
\hline \multicolumn{2}{|l|}{ Site 3} \\
\hline crRNA' & 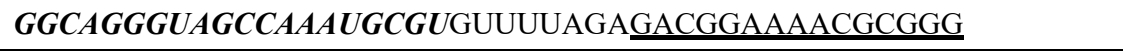 \\
\hline tracrRNA' & $\begin{array}{l}\text { UGCGAUCAAACUGCCAAGUUAAAAUAAGGCUAGUCCGUUAUCAACUUGA } \\
\text { AAAAGUGGCACCGAGUCGGUGCU }\end{array}$ \\
\hline soxS-sgRNA & $\begin{array}{l}\boldsymbol{A} \boldsymbol{U} \boldsymbol{C} \boldsymbol{C} \boldsymbol{G} \boldsymbol{A} \boldsymbol{A} \boldsymbol{U} \boldsymbol{A} \boldsymbol{A} \boldsymbol{U} \boldsymbol{U} \boldsymbol{U} \boldsymbol{U} \boldsymbol{C U} \boldsymbol{G} \boldsymbol{A} \boldsymbol{U} G U U U U A G A G C U A G A A A U A G C A A G U U A A A A \\
\text { UAAGGCUAGUCCGUUAUCAACUUGAAAAAGUGGCACCGAGUCGGUGCU }\end{array}$ \\
\hline \multicolumn{2}{|l|}{ Control } \\
\hline crRNA' & GGCAGGGUAGCCAAAUGCGUGUUUAGACCUCCUCGCCCUUGC \\
\hline tracrRNA' & $\begin{array}{l}\text { UGAUGGCCAUGUUAUAAGUUAAAAUAAGGCUAGUCCGUUAUCAACUUGA } \\
\text { AAAAGUGGCACCGAGUCGGUGCU }\end{array}$ \\
\hline
\end{tabular}

Table S6. RNA sequences used in Figure 5.

\begin{tabular}{|c|c|}
\hline & RNA sequence (5'-3') \\
\hline thyA-crRNA-14 & $\boldsymbol{A} \boldsymbol{A} \boldsymbol{U} \boldsymbol{G} \boldsymbol{G A \boldsymbol { A } A \boldsymbol { G } \boldsymbol { C } \boldsymbol { G } \boldsymbol { U } \boldsymbol { C } \boldsymbol { C } \boldsymbol { G } \boldsymbol { G } \boldsymbol { U } \boldsymbol { U } \boldsymbol { C G U U U U A G A G C U A U G C U G U U U U G ~ }}$ \\
\hline Null-crRNA & GUUUUAGAGCUAUGCUGUUUUG \\
\hline tracrRNA-14 & $\begin{array}{l}\text { CAAAACAGCAUAGCAAGUUAAAAUAAGGCUAGUCCGUUAUCAACUUGAAA } \\
\text { AAGUGGCACCGAGUCGGUGCU }\end{array}$ \\
\hline \multicolumn{2}{|l|}{ Exogenous RNA } \\
\hline thyA-crRNA' & $\boldsymbol{A} \boldsymbol{A} \boldsymbol{U} \boldsymbol{G} \boldsymbol{G} \boldsymbol{A} \boldsymbol{A} \boldsymbol{A} \boldsymbol{G} \boldsymbol{C} \boldsymbol{G} \boldsymbol{U} \boldsymbol{U} \boldsymbol{C C G} \boldsymbol{C} \boldsymbol{U} U \boldsymbol{C}$ GUUUUAGAAGUUUGCGCAACGUU \\
\hline tracrRNA' & $\begin{array}{l}\text { CCGAGUGGGACUUAUAAGUUAAAAUAAGGCUAGUCCGUUAUCAACUUGAA } \\
\text { AAAGUGGCACCGAGUCGGUGCU }\end{array}$ \\
\hline Trigger(T) & AACGUUGCGCAAACUAUAAGUCCCACUCGG \\
\hline $\mathrm{T}_{\mathrm{w}}$ & UUACACUGAUGGGGCGAUCGGUGCCCAUCA \\
\hline soxS Site 1 & \\
\hline
\end{tabular}




\begin{tabular}{|c|c|}
\hline thyA-crRNA' & $\boldsymbol{A} \boldsymbol{A} \boldsymbol{U} \boldsymbol{G} \boldsymbol{G A A} \boldsymbol{A} \boldsymbol{G C G U U \boldsymbol { C C G } \boldsymbol { G } U \boldsymbol { U } \boldsymbol { C G U U U U A G A U G C G A U A A G A U C C U G }}$ \\
\hline \multirow{2}{*}{ tracrRNA' } & $\underline{\text { AUGCUCGUCAAUCCAAAGUUAAAAUAAGGCUAGUCCGUUAUCAACUUGAA }}$ \\
\hline & AAAGUGGCACCGAGUCGGUGCU \\
\hline \multicolumn{2}{|l|}{ Control } \\
\hline crRNA' & AAUGGAAAGCGUUCCGGUUCGUUUUAGAGAACCGGAACGCGUU \\
\hline \multirow{2}{*}{ tracrRNA' } & $\underline{\text { CCGAGUGGGACUUAUAAGUUAAAAUAAGGCUAGUCCGUUAUCAACUUGAA }}$ \\
\hline & AAAGUGGCACCGAGUCGGUGCU \\
\hline
\end{tabular}

Table S7. RNA sequences used in Figure S14 and Figure S15.

\begin{tabular}{|c|c|}
\hline & RNA sequence (5'-3') \\
\hline \multicolumn{2}{|l|}{ ASCL1-gRNA-d14 } \\
\hline crRNA-14 & $\boldsymbol{U} \boldsymbol{G} \boldsymbol{G} \boldsymbol{A} \boldsymbol{G} \boldsymbol{A} \boldsymbol{G} \boldsymbol{U} \boldsymbol{U} \boldsymbol{U} \boldsymbol{G C A} \boldsymbol{A} \boldsymbol{G} \boldsymbol{G} \boldsymbol{A} \boldsymbol{G} \boldsymbol{C G U U U U A G A G C U A U G C U G U U U U G ~}$ \\
\hline tracrRNA14 & $\begin{array}{l}\text { CAAAACAGCAUAGCAAGUUAAAAUAAGGCUAGUCCGUUAUCAACUUGAA } \\
\text { AAAGUGGCACCGAGUCGGUGCU }\end{array}$ \\
\hline \multicolumn{2}{|l|}{ ASCL1-gRNA-d10 } \\
\hline crRNA-10 & $\boldsymbol{U} \boldsymbol{G G} \boldsymbol{A} \boldsymbol{G} \boldsymbol{A} \boldsymbol{G} \boldsymbol{U} \boldsymbol{U} \boldsymbol{U} \boldsymbol{G C A \boldsymbol { A } \boldsymbol { G } \boldsymbol { A } \boldsymbol { A } \boldsymbol { G } \boldsymbol { C G U U U U A G A G C U A U G C U G U }}$ \\
\hline tracrRNA10 & $\begin{array}{l}\text { ACAGCAUAGCAAGUUAAAAUAAGGCUAGUCCGUUAUCAACUUGAAAAAG } \\
\text { UGGCACCGAGUCGGUGCU }\end{array}$ \\
\hline \multicolumn{2}{|l|}{ ASCL1-gRNA-d6 } \\
\hline crRNA-6 & 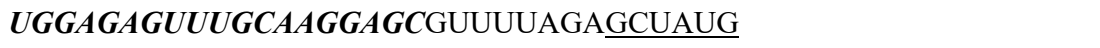 \\
\hline tracrRNA-6 & $\begin{array}{l}\text { CAUAGCAAGUUAAAAUAAGGCUAGUCCGUUAUCAACUUGAAAAAGUGGC } \\
\text { ACCGAGUCGGUGCU }\end{array}$ \\
\hline \multicolumn{2}{|l|}{ ASCL1-gRNA-d5 } \\
\hline crRNA-4 & 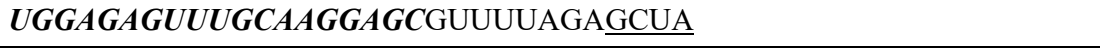 \\
\hline tracrRNA-4 & $\begin{array}{l}\text { UAGCAAGUUAAAAUAAGGCUAGUCCGUUAUCAACUUGAAAAAGUGGCAC } \\
\text { CGAGUCGGUGCU }\end{array}$ \\
\hline \multicolumn{2}{|l|}{ ASCL1-gRNA-d4 } \\
\hline crRNA-3 & 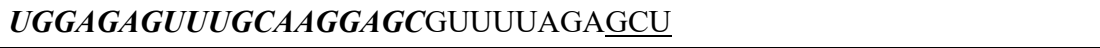 \\
\hline tracrRNA-3 & $\begin{array}{l}\text { AGCAAGUUAAAAUAAGGCUAGUCCGUUAUCAACUUGAAAAAGUGGCACC } \\
\text { GAGUCGGUGCU }\end{array}$ \\
\hline \multicolumn{2}{|l|}{ ASCL1-gRNA-d3 } \\
\hline crRNA-2 & 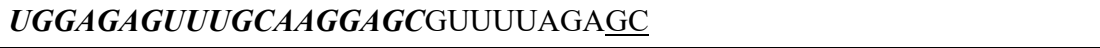 \\
\hline tracrRNA-2 & $\begin{array}{l}\text { GCAAGUUAAAAUAAGGCUAGUCCGUUAUCAACUUGAAAAAGUGGCACCG } \\
\text { AGUCGGUGCU }\end{array}$ \\
\hline \multicolumn{2}{|l|}{ ASCL1-gRNA-d2 } \\
\hline crRNA-1 & 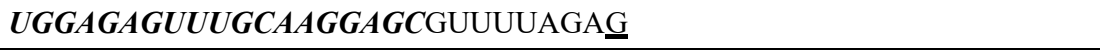 \\
\hline tracrRNA-1 & 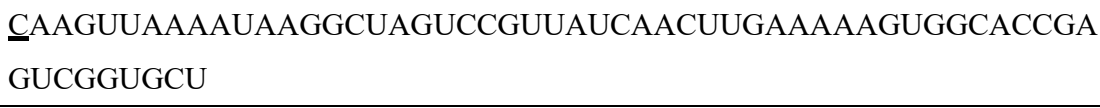 \\
\hline \multicolumn{2}{|l|}{ ASCL1-gRNA-d0 } \\
\hline crRNA-0 & 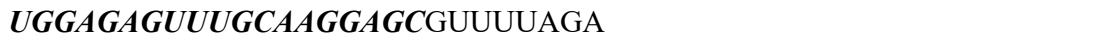 \\
\hline tracrRNA-0 & AAGUUAAAAUAAGGCUAGUCCGUUAUCAACUUGAAAAAGUGGCACCGAG \\
\hline
\end{tabular}




\begin{tabular}{|c|c|}
\hline & UCGGUGCU \\
\hline \multicolumn{2}{|l|}{$\mathbf{A}$} \\
\hline crRNA' & $\begin{array}{l}\boldsymbol{U} \boldsymbol{G G} \boldsymbol{A} \boldsymbol{G} \boldsymbol{A} \boldsymbol{G} \boldsymbol{U} \boldsymbol{U} \boldsymbol{U} \boldsymbol{G C A} \boldsymbol{A} \boldsymbol{G} \boldsymbol{G} \boldsymbol{A} \boldsymbol{G} \boldsymbol{C G U U U U A G A G C U A G U U U G C G C A A C G U U G U U G C} \\
\underline{\mathrm{C}}\end{array}$ \\
\hline tracrRNA' & $\begin{array}{l}\text { CUCGAUCCGAGUGGGACUUAUAGCAAGUUAAAAUAAGGCUAGUCCGUUA } \\
\text { UCAACUUGAAAAAGUGGCACCGAGUCGGUGCU }\end{array}$ \\
\hline Trigger & GGCAACAACGUUGCGCAAACUAUAAGUCCCACUCGGAUCGAGC \\
\hline \multicolumn{2}{|l|}{ B } \\
\hline crRNA-14 & $\boldsymbol{G} \boldsymbol{G} \boldsymbol{G} \boldsymbol{G} \boldsymbol{G} \boldsymbol{A} \boldsymbol{G} \boldsymbol{U} \boldsymbol{U} \boldsymbol{A} \boldsymbol{G} \boldsymbol{G} \boldsymbol{G} \boldsymbol{A} \boldsymbol{G} \boldsymbol{U} \boldsymbol{G} \boldsymbol{G} \boldsymbol{G} \mathrm{GUUUUAGAGCUAUGCUGUUUUG}$ \\
\hline crRNA' & 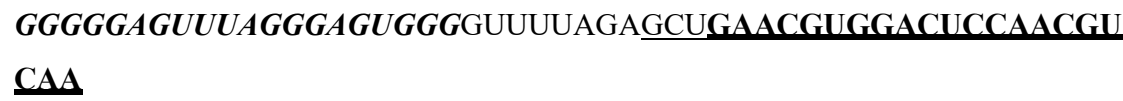 \\
\hline tracrRNA' & $\begin{array}{l}\text { CUGAUAGACGGUUUUUCGCCCAGCAAGUUAAAAUAAGGCUAGUCCGUUA } \\
\text { UCAACUUGAAAAAGUGGCACCGAGUCGGUGCU }\end{array}$ \\
\hline Trigger & UUGACGUUGGAGUCCACGUUCGGGCGAAAAACCGUCUAUCAG \\
\hline $\mathrm{T}_{\mathrm{w}}$ & UAUUAGCGCCGGGUUGUAAAACGACCGGCGCUAAUAUUCACC \\
\hline
\end{tabular}

Table S8. qPCR primers used in this study.

\begin{tabular}{|c|c|c|}
\hline Gene & F-primer (5'-3') & R-primer (5'-3') \\
\hline rssA & AGGACTCATGGCACCTGTTG & ATGCACGCGTGAGGGAAATA \\
\hline lacZ & GCCCATCTACACCAACGTGA & TGAGCGAGTAACAACCCGTC \\
\hline galA & ATTTGGCTACCCTGCCACTC & GCGAACTTTACGGTCATCGC \\
\hline mCherry & TTCATGTACGGCTCCAAGGC & CTGCTTGATCTCGCCCTTCA \\
\hline oxyR & AGATTCCTGCCACGTTAGCC & GGTAGCAGGTACGGTCCAAC \\
\hline soxR & ATCCGTAACAGCGGCAATCA & ACATCCGTCCAGTTCGTCAC \\
\hline soxS & TCGCATGGATTGACGAGCAT & CTGCTGCGAGACATAACCCA \\
\hline thyA & ACGCATCTGTACAGCAACCA & ATAGCCACCGGCGCTTTAAT \\
\hline $16 \mathrm{~S}$ RNA & TAATACGGAGGGTGCAAGCG & CTTCGCCACCGGTATTCCTC \\
\hline micF & GCTATCATCATTAACTTTATTTA & AAACCGGATGCGAGGCATC \\
\hline sgrS & AAGTTGTGCTGGTTGCGTTG & GCTGGCGGGTGATTTTACAC \\
\hline oxyS & AGCGGCACCTCTTTTAACCC & CGGATCCTGGAGATCCGCAA \\
\hline GAPDH & ACAGTCAGCCGCATCTTCTT & ACGACCAAATCCGTTGACTC \\
\hline ASCL1 & GGAGCTTCTCGACTTCACCA & AACGCCACTGACAAGAAAGC \\
\hline
\end{tabular}




\section{Supporting Figures}

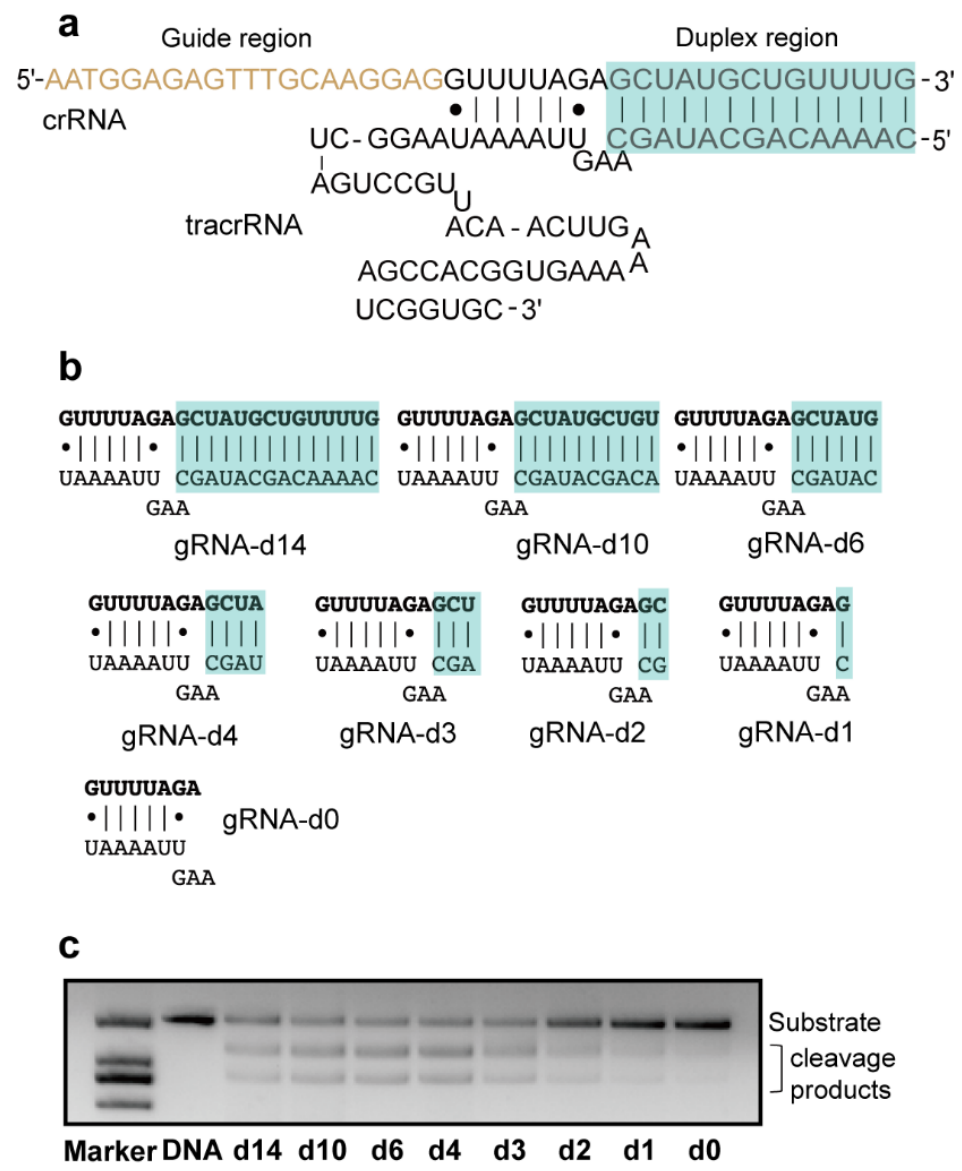

Figure S1. The effect of truncated crRNA/tracrRNA on the cleavage activity of CRISPR/Cas9. (a) The structure of natural duplex formed between crRNA and tracrRNA. The duplex region outside the binding pocket of Cas9 is marked with the cyan color. (b) The gradually reduced duplex formed between crRNA and tracrRNA from 14-bp to 0-bp. The altered duplex segments are marked with the cyan color. (c) Biochemical cleavage activities of Cas9 with truncated crRNA/tracrRNA in the presence of different lengths of the binding duplex. Cleaved DNA products were shown from the relatively fast-migrated bands. 
a
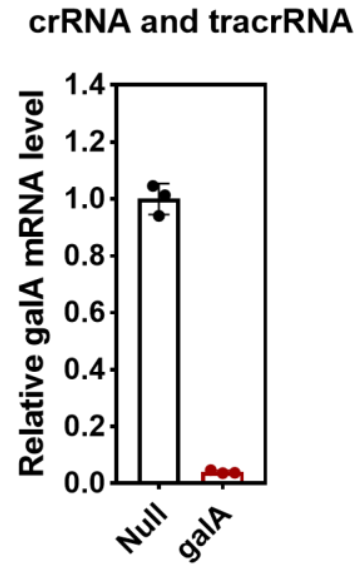

b Single gRNA

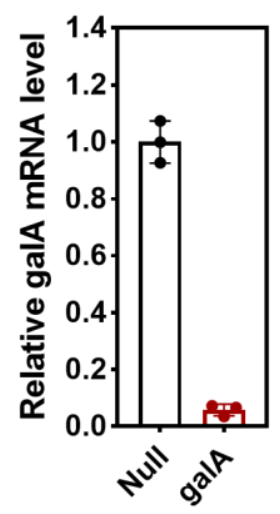

Figure S2. The regulatory efficiency of crRNA-tracrRNA and single gRNA-based CRIPSRi in E. coli. (a) The galA gene expression was sharply knocked down by the crRNA/tracrRNA-based CRISPRi to reach $\sim 4 \%$. (b) The inhibition of galA expression reached $\sim 6 \%$ by the single gRNA (sgRNA)-based CRISPRi. "Null" indicated the gRNA that could not target any gene site in the cells. 

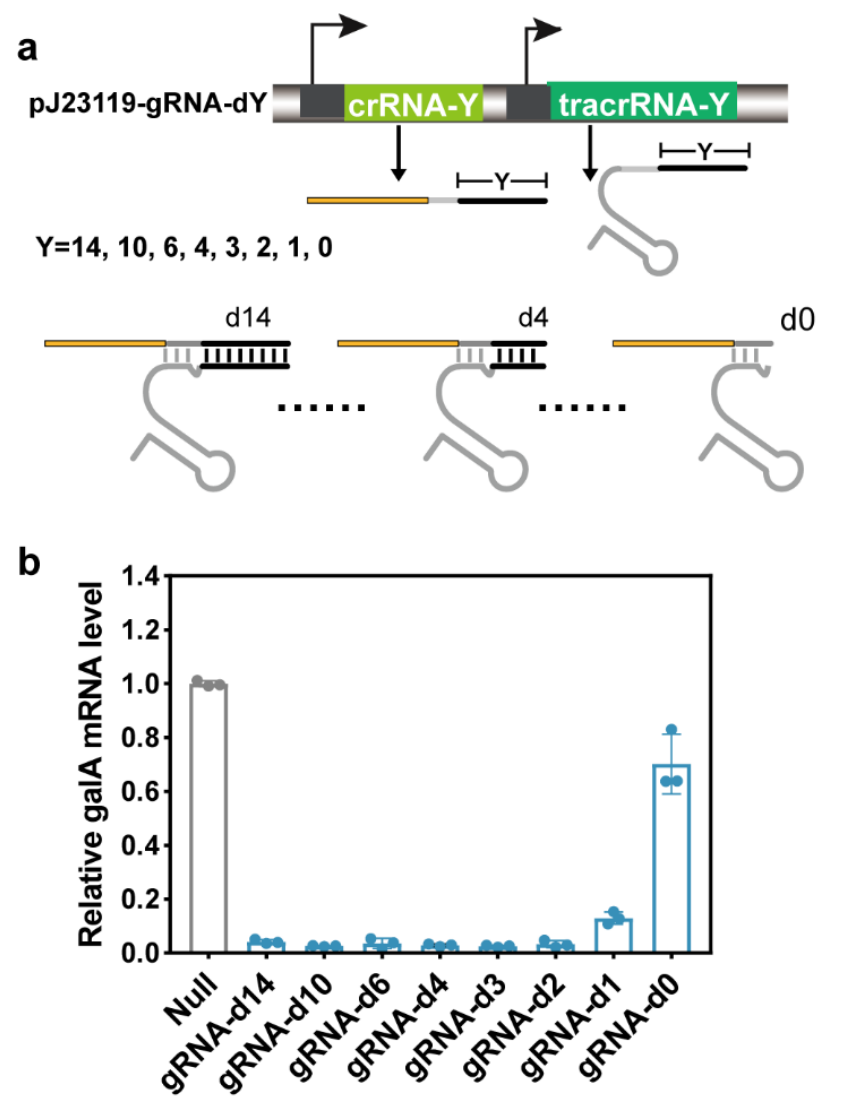

Figure S3. Impacts of the truncated crRNA/tracrRNA on the CRISPRi efficiency in E. coil. (a) Sketch for the duplex of truncated crRNAs and tracrRNAs. The numbers 14, 10, 6, 4, 3, 2, 1 and 0 represented the length of base pairing between the truncated crRNA and tracrRNA. (b) The expression of galA level under different truncated gRNAs. Clearly, when the binding duplex between the truncated crRNA and tracrRNA was reduced to 0-bp, the CRISPRi effect on the endogenous galA gene was significantly abolished. Error bars were standard deviations derived from three biological replicates. "Null" indicated the gRNA that could not target any gene site in the cells. 

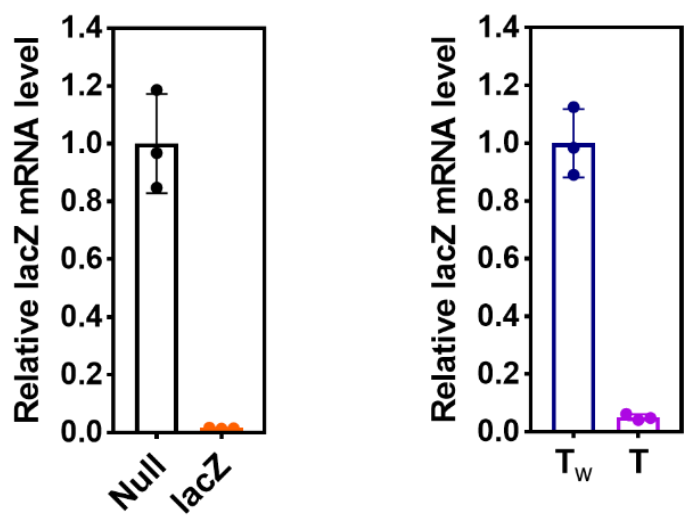

Figure S4. An independent exogenous RNA to control the expression of endogenous gene lacZ in E. coli. In the left panel, the CRISPRi effect on lacZ expression by the natural assembly of gRNA was shown as comparison. Naturel assembly of gRNA without the target region was selected as control (Null). The activable CRISPRi effect by the trigger RNA (T) with the 15-bp binding duplex was presented in the right panel. As a control, an incorrect trigger RNA $\left(\mathrm{T}_{\mathrm{w}}\right)$ was introduced to replace the trigger RNA. Error bars were standard deviations derived from three biological replicates. 
a

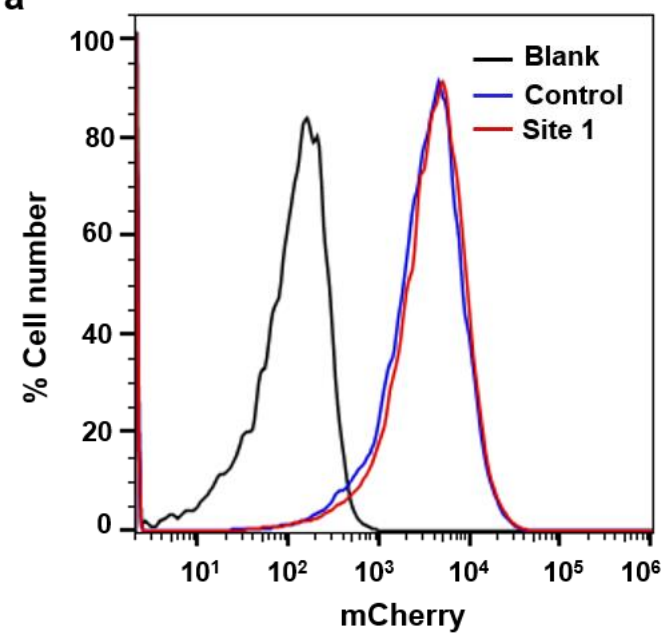

b

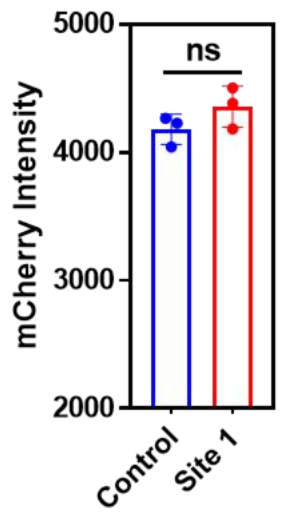

Figure S5. Introduction of the binding crRNA'/tracrRNA' could hardly affect the translation of the mCherry mRNA based on the flow cytometry analysis. (a) Representative profiles of flow cytometry. (b) Quantitative analysis of the average fluorescence of mCherry. Blank: E. coli cells without mCherry; Control: plasmids expressing non-binding crRNA'/tracrRNA', dCas9 and mCherry were transformed into E. coli cells; Site 1: plasmids expressing the corresponding crRNA $^{\prime} /$ tracrRNA', dCas9 and mCherry were transformed into E. coli cells as described in Figure $2 \mathrm{e}$, in which the mCherry mRNA bound with the corresponding crRNA'/tracrRNA' to form the active gRNA'. "ns": not significant (unpaired $t$ test, $n=3$ ). 

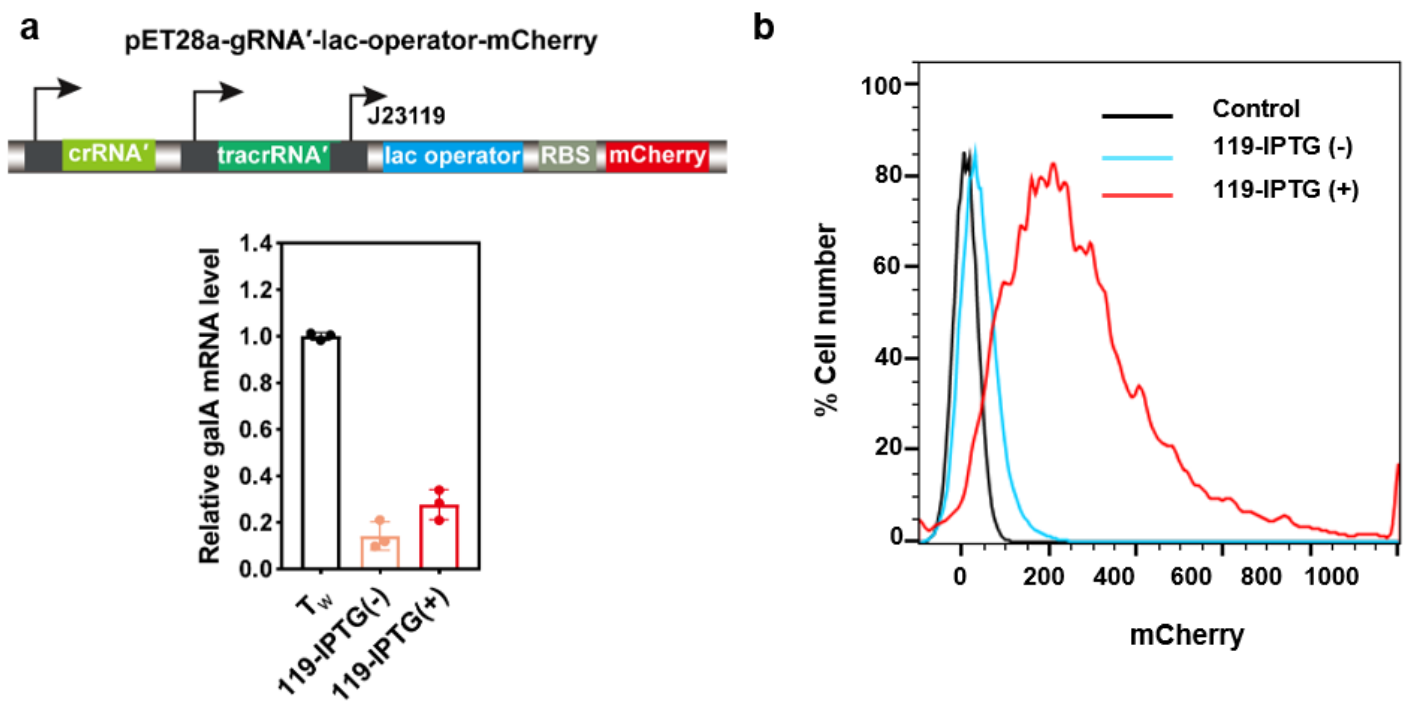

Figure S6. Regulation of the galA level through controllable mCherry expressions via the lac operator. (a) The plasmid construct and the CRISPRi effects on galA. Under the strong promoter J23119, either the inhibited expression or the IPTG-induced expression of mCherry mRNA could both trigger the potent CRISPRi effect on galA level. (b) Flow cytometry before and after IPTG $(400 \mu \mathrm{M})$ induction. Cells without the introduction of the mCherry gene were selected as control. Cell numbers were normalized. Error bars were standard deviations derived from three biological replicates. 
a

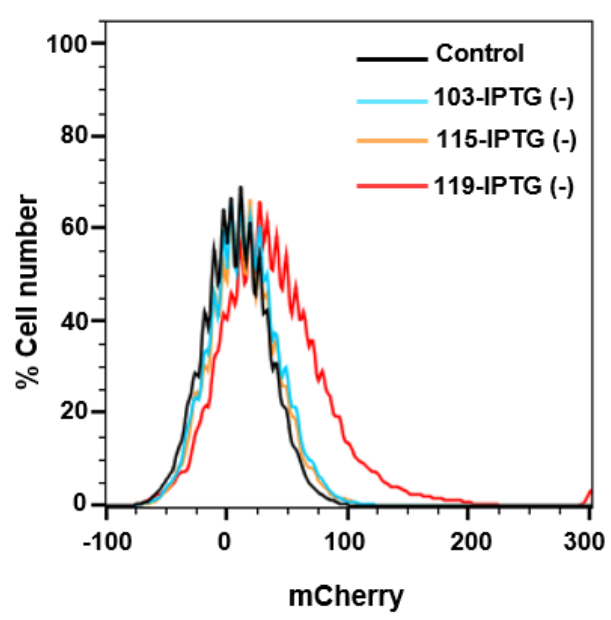

C

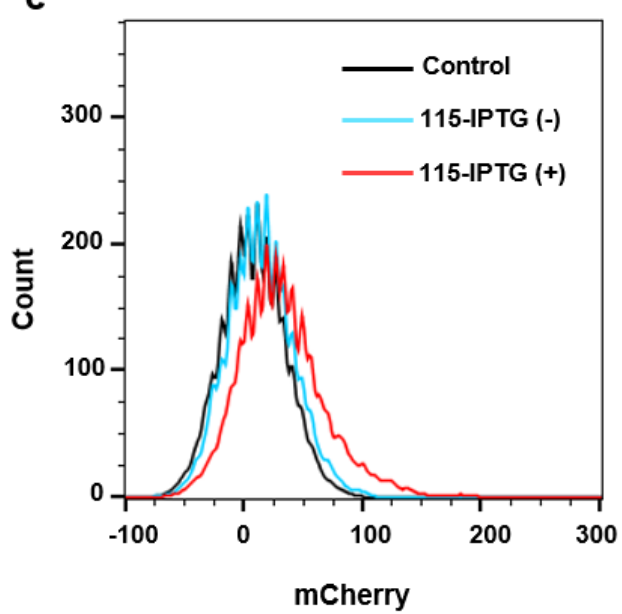

b

pET28a-gRNA'-lac-operator-mCherry
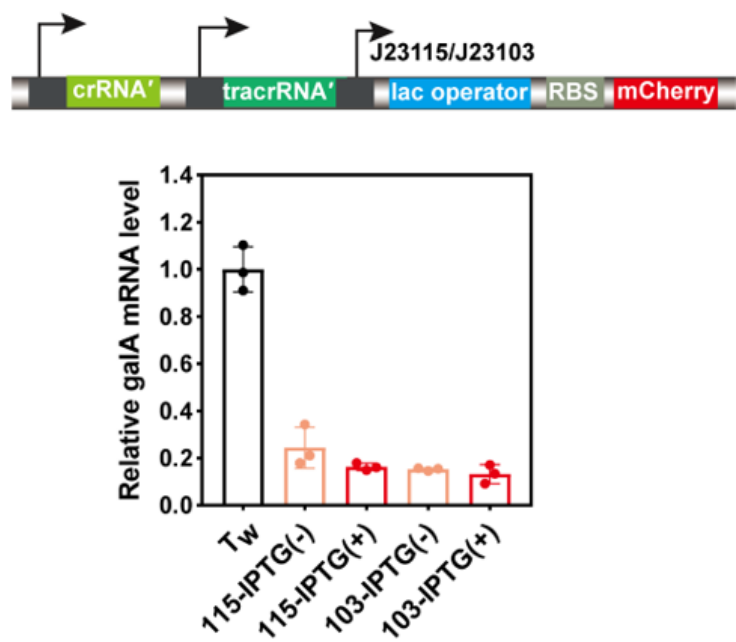

d

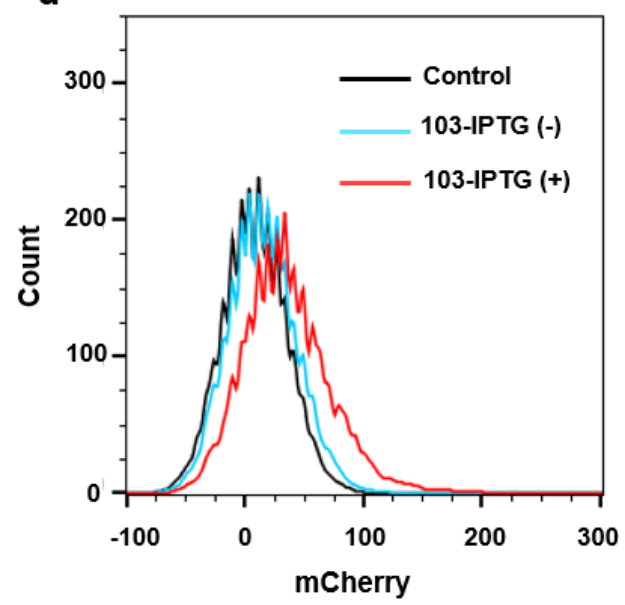

Figure S7. Regulation of the galA level through induced expressions of mCherry under the control of weak promotors. (a) Reduced the expressional leakage under the control of the weak promoters J23115 and J23103 compared to strong promoter J23119 in the presence of lac repressor. Cell numbers are normalized to mode. (b) Under the control of the weak promoters J23115 and J23103, the mCherry mRNA could also greatly trigger the galA knock-down with or without IPTG induction. A non-mCherry RNA was expressed to replace the mCherry mRNA as the control $\left(\mathrm{T}_{\mathrm{w}}\right)$. (c) and (d) demonstrated the effectiveness of IPTG-induced expressions of mCherry under the control of the weak promoters J23115 and J23103, even though the mCherry intensities were relatively low. Cells without introduction of the mCherry gene were selected as control. Error bars were standard deviations derived from three biological replicates. 


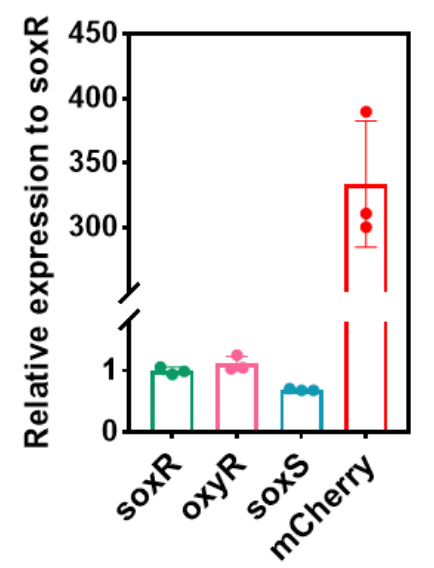

Figure S8. Relative expressional levels of the exogenous mCherry compared with endogenous mRNAs in E. coil. The oxyR, soxS, and mCherry mRNA levels were normalized to soxR. The mCherry mRNA was introduced by the plasmid under the control of the J23103 promotor with the lac repressor. Error bars were standard deviations derived from three biological replicates.

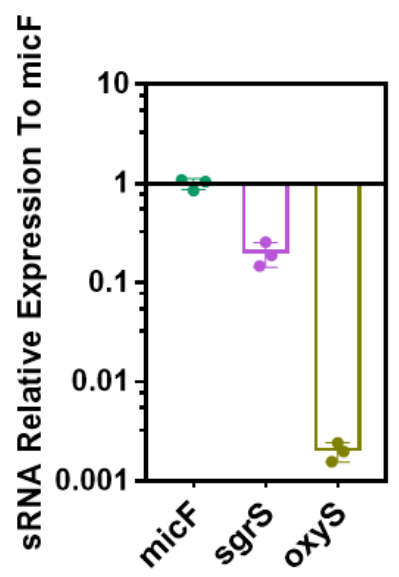

Figure S9. Relative sRNA expression levels of micF, sgrS, and oxyS in E. coil. The expression levels of sgrS and oxyS were normalized to micF. Error bars were standard deviations derived from three biological replicates. 

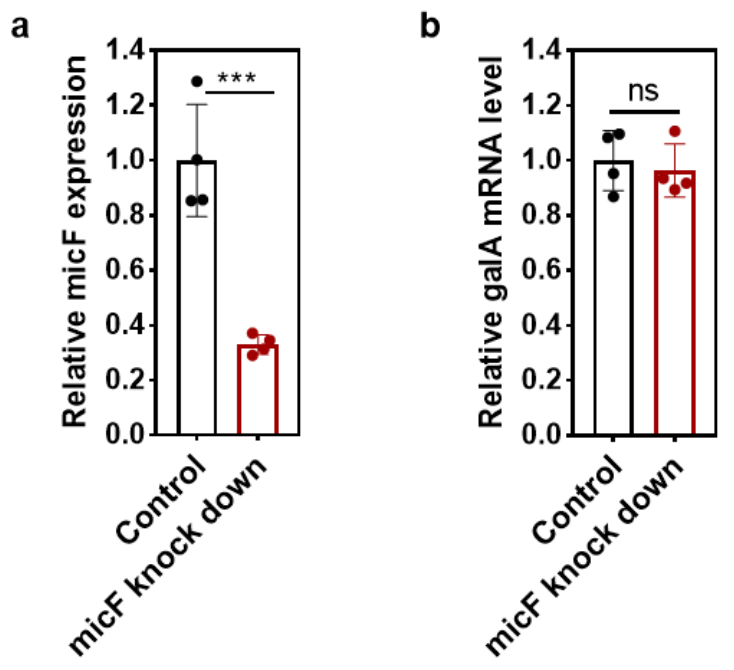

Figure S10. Failure of the induced regulation between the galA and micF genes without the correct crRNA'/tracrRNA'. (a) Knockdown effect of the micF gene by CRISPRi in E. coli. (b) Knockdown of micF cannot affect the galA RNA expression with incorrect crRNA'/tracrRNA'. The only difference between Figure 3d and this figure was an alteration of the crRNA'/tracrRNA', with which the micF RNA could not form the ternary assembly. The control indicated the samples without micF knockdown. "ns": not significant; $* * * P<0.001$ (unpaired t test, two-tailed $P$ value, $n=4)$. The plasmid information was listed in Table S1. Data were shown as mean values with standard deviations derived from four biological replicates. 

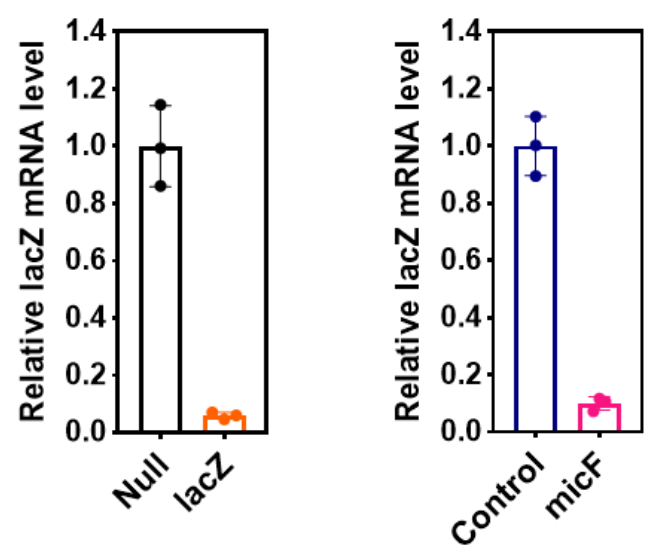

Figure S11. The lacZ gene could also be linked with micF by the corresponding crRNA'/tracrRNA'. The CRISPRi effect on the lacZ expression by the natural assembly of gRNA was shown as comparison in the left panel. Transcriptional inhibition of the lacZ gene induced by micF was shown in the right panel. The crRNA'/tracrRNA' that shared the same guide sequence against the lacZ gene but could not form the ternary assembly with micF was selected as control. Data were shown as mean values with standard deviations derived from three biological replicates. 


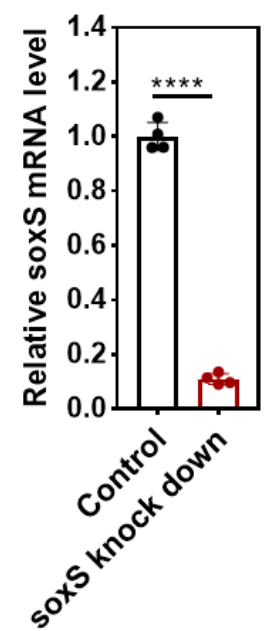

b

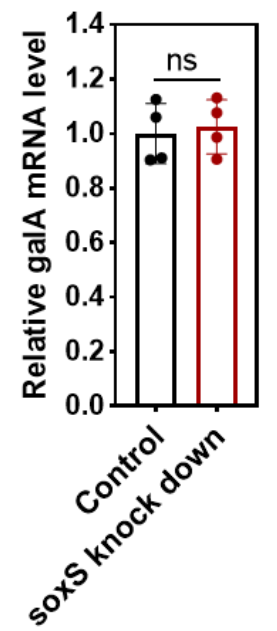

Figure S12. Failure of the induced regulation between the galA and soxS genes without the correct crRNA'/tracrRNA'. (a) Knockdown effect of the soxS gene by CRISPRi in E. coli. (b) Knockdown of soxS cannot affect the galA RNA expression with incorrect crRNA'/tracrRNA'. The only difference between Figure $4 \mathrm{c}$ and this figure was an alteration of the crRNA'/tracrRNA', with which the soxS RNA could not form the ternary assembly. The control indicated the samples without soxS knockdown. "ns": not significant; $* * * * P<0.0001$ (unpaired t test, two-tailed $P$ value, $\mathrm{n}=4$ ). The plasmid information was listed in Table S1. Data were shown as mean values with standard deviations derived from four biological replicates. 
a

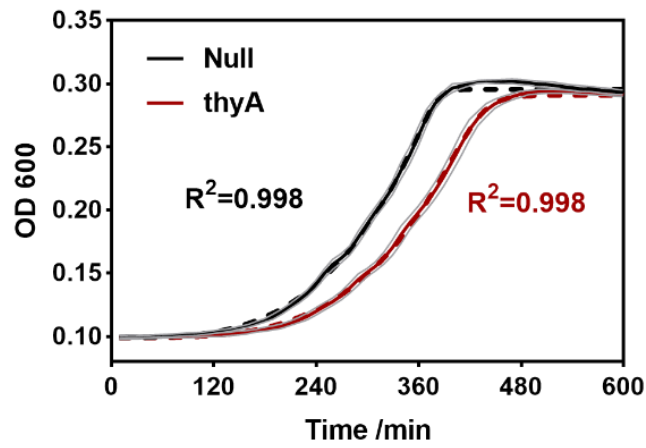

b

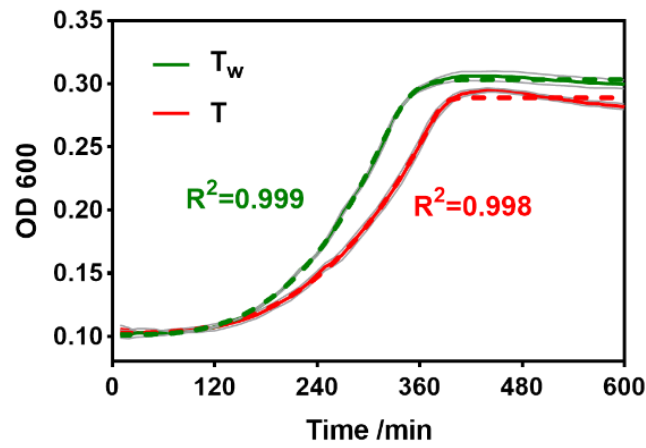

C

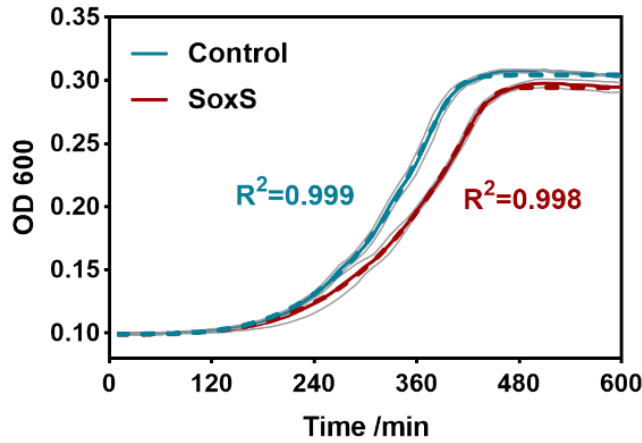

Figure S13. Fitting of the growth curves of E. coli. (a) Growth curves of E. coil with or without knockdown of thyA. (b) Growth curves of E. coli with the cognate trigger RNA (T) or the incorrect one $\left(\mathrm{T}_{\mathrm{w}}\right.$ ) to the crRNA'/tracrRNA'. (c) Growth curves of E. coli with (soxS) or without (control) the soxS-induced knockdown of thyA. Solid lines are experimentally measured curves; dashed lines are obtained from the mathematical model presented in Materials and Methods, with parameters fitted against these experimental data. $\mathrm{R}^{2}$ shows the goodness of fitting. 

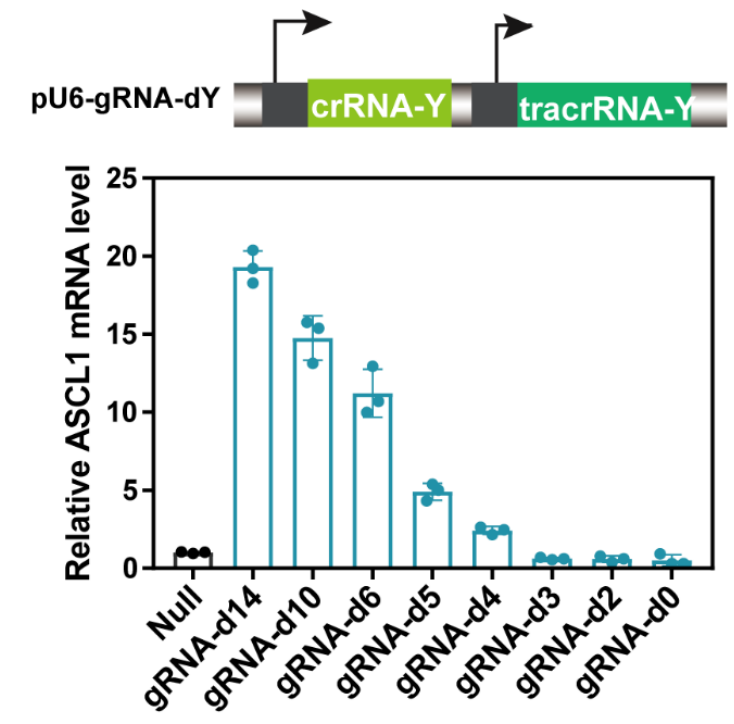

Figure S14. The truncation of the binding duplex between crRNA and tracrRNA affected the efficiency of CRISPRa on the ASCL1 mRNA expression in HEK-293T cells. The expression level of ASCL1 under different truncated gRNAs. Clearly, when the binding duplex between crRNA and tracrRNA was reduced to 3-bp, CRISPRa effect on the endogenous ASCL1 gene was completely abolished. The numbers 14, 10, 6, 5,4, 3, 2, 1 and 0 represented the length of base pairing between the crRNA and tracrRNA. The crRNA without the target region was selected as control (Null). Data were shown as mean values with standard deviations derived from three biological replicates. 

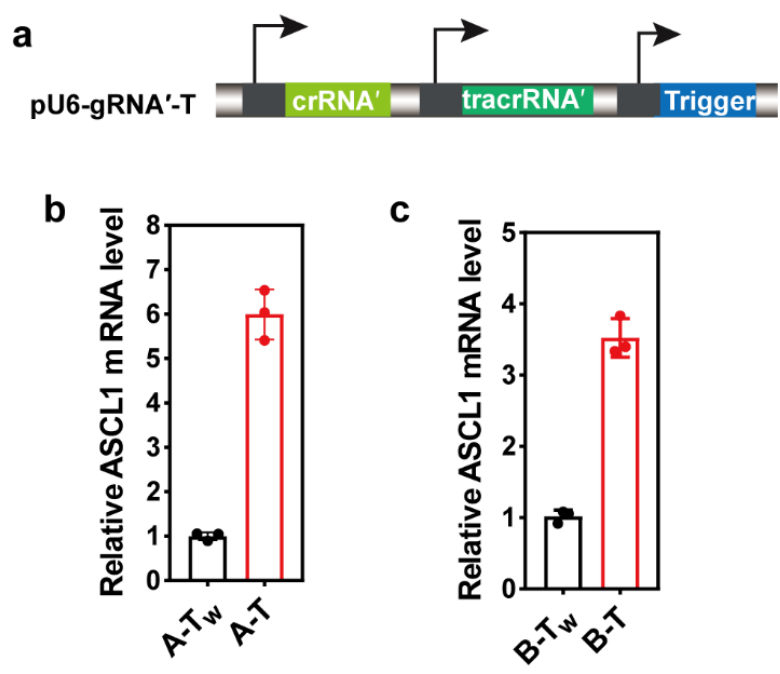

Figure S15. Control of endogenous ASCL1 gene expression by exogenous RNAs induced ternary assembly of gRNA' in HEK-293T cells. (a) The plasmid with the crRNA'/tracrRNA' and the trigger RNA under three independent U6 promoters. (b) Activable CRISPRa effects on ASCL1 by the trigger RNA-A and the corresponding crRNA'/tracrRNA'. (c) Control of ASCL1 mRNA expressions by another sequence of trigger RNA-B and the corresponding crRNA'/tracrRNA'. An incorrect trigger RNA $\left(\mathrm{T}_{\mathrm{w}}\right)$ was introduced to replace the cognate trigger RNA as control. Data were shown as mean values with standard deviations derived from three biological replicates.

\section{References:}

(1) Li, S.; Jendresen, C. B.; Grünberger, A.; Ronda, C.; Jensen, S. I.; Noack, S.; Nielsen, A. T., Enhanced protein and biochemical production using CRISPRi-based growth switches. Metabolic engineering 2016, 38, 274-284.

(2) Lin, J.; Liu, Y.; Lai, P.; Ye, H.; Xu, L., Conditional guide RNA through two intermediate hairpins for programmable CRISPR/Cas9 function: building regulatory connections between endogenous RNA expressions. Nucleic Acids Res 2020, 48, 11773-11784. 